\title{
Population growth affects intrinsic and extrinsic noise in gene expression
}

Philipp Thomas ${ }^{1}$

Department of Mathematics, Imperial College London, London SW7 2AZ, $U K \mathrm{a})$

Clonal cells of exponentially growing populations vary substantially from cell to cell. The main drivers of this heterogeneity are the population dynamics and stochasticity in the intracellular reactions, which are commonly studied separately. Here we develop an agent-based framework that allows tracking of the biochemical dynamics in every single cell of a growing population that accounts for both of these factors. Apart from the common intrinsic variability of the biochemical reactions, the framework also predicts extrinsic noise arising from fluctuations in the histories of cells without the need to introduce fluctuating rate constants. Instead, these extrinsic fluctuations are explained by cell cycle fluctuations and differences in cell age, which are ubiquitously observed in growing populations. We give explicit formulas to quantify mean molecule numbers, intrinsic and extrinsic noise statistics as measured in two-colour experiments. We find that these statistics may differ significantly depending on the experimental setup used to observe the cells. We illustrate this fact using (i) averages over an isolated cell lineage tracked over many generations as observed in the mother machine, (ii) snapshots of a growing population with known cell ages as recorded in time-lapse microscopy, and (iii) snapshots of unknown cell ages as measured from static images. Our integrated approach applies to arbitrary biochemical networks and generation time distributions. By employing models of stochastic gene expression and feedback regulation, we elucidate that isolated lineages, as compared to snapshot data, can significantly overestimate the mean number of molecules, overestimate extrinsic noise but underestimate intrinsic noise and have qualitatively different sensitivities to cell cycle fluctuations.

\footnotetext{
a)Electronic mail: p.thomas@imperial.ac.uk
} 


\section{Introduction}

The behaviour of clonal cells varies substantially from cell to cell and over time ${ }^{12}$. Iden- 2 tifying the sources of these fluctuations can help us to understand how clonal cells diversify 3 their responses and to reveal the function of gene circuits and signalling networks. For their 4 quantification, it is often convenient to break down the experimentally observed variability 5 into functional components. Commonly one wishes to separate fluctuations inherent in the 6 circuit dynamics itself, called intrinsic noise, from fluctuations that arise from embedding 7 the circuit in the environment of the cell, called extrinsic noise.

A possible resolution to this problem is to place and simultaneously measure a second 9 independent copy of the circuit in the cell, as has been done in E. $\operatorname{col}^{1}$, yeast ${ }^{3}$, mammalian 10 cells ${ }^{4}$ and plants ${ }^{5}$. The difference between the two circuit copies measures the intrinsic noise ${ }_{11}$ whereas their correlations measure the extrinsic noise component. Intrinsic noise arises from 12 the random nature of the involved biochemical reactions. Extrinsic noise originates from fac- ${ }_{13}$ tors affecting both circuits in the same way. These can, for instance, be modelled by reaction ${ }_{14}$ rates that fluctuate between cells or over time due to shared resources, promoter architecture 15 or upstream pathways. Such sources of extrinsic noise have been studied extensively in the ${ }_{16}$ literature 6 [14.

A less commonly studied but equally important source of extrinsic noise is the population 18 dynamics 15 . Since intracellular molecule numbers must double over the cell division cycle, 19 a two-fold variation of expression levels is expected from cell proliferation alone. Moreover, 20 the cell cycle itself is subject to tremendous variation providing an additional source of ${ }_{21}$ extrinsic variability. For example, generation times in Escherichia coli ${ }^{16}$, budding yeast ${ }_{22}^{17}$ and mammalian cells ${ }^{18}$ vary about $30-50 \%$. These sources should therefore prevail in ${ }_{23}$ growing cells, populations and tissues.

Modelling approaches for understanding the effects of the cell cycle on gene expression 25 noise are only recently being developed ${ }^{19} 24$. These studies are often restricted to a single ${ }_{26}$ isolated cell observed over successive cell divisions and measuring variability over time, sim- $\quad 27$ ilar to a lineage in the mother machine ${ }^{25}$. Many experiments, however, report cell-to-cell 28 variability across snapshots of an exponentially growing cell population. These approaches 29 either use time-lapse microscopy ${ }^{26 / 27}$ or analyse snapshots with distributed cell ages as ob- 30 served in flow cytometry, smFISH or similar techniques 28 
Recent studies elucidated that population snapshots and lineages can significantly deviate 32 from each other in their statistics $24 \mid 31$. To-date, however, there exists no general analytical 33 framework with which to quantify the gene expression fluctuations in populations. We are ${ }_{34}$ thus lacking the means with which to understand, compare and integrate the knowledge from 35 different experiments such as mother machines, time-lapse microscopy or fixed-cell images. 36 Agent-based approaches allowing to track the expression levels of each individual cell in a 37 growing population are ideally suited to address this issue.

In this manuscript, we develop such an approach to characterise the statistics of bio- 39 chemical reaction networks in a growing and dividing cell population. In this framework, an 40 agent is represented by a cell whose biochemical decomposition changes due to stochastic ${ }_{41}^{4}$ reaction kinetics and cell divisions. In Sec. II A we show how to analytically characterise the ${ }_{42}$ joint distribution of cell age and molecule content per cell in a snapshot of the population. ${ }^{43}$ We then, in Sec. IIB, derive the exact moment equations of this model. 44

Since stochastic models are rarely solvable, we propose an analytically tractable approx- $\quad 45$ imation to mean and covariances in Sec. IIIA. Intrinsic and extrinsic noise sources as they 46 are measured using two-reporter systems are in-built in the agent-based approach, and we ${ }_{47}$ explain how to decompose the apparent noise into the respective components. We further ${ }_{48}$ elaborate on the decomposition in cases where the cell age is unknown, a situation com- ${ }_{49}$ monly encountered when analysing data from population snapshots or flow cytometry. We 50 demonstrate how to practically compute the noise decomposition in Sec. IIIB, illustrate the ${ }_{51}$ results using a simple two-reporter system, and study how circuit dynamics can be tuned to ${ }_{52}$ suppress either intrinsic or extrinsic fluctuations.

\section{Methods}

We model the dynamics of a dividing population of cell agents. The state of each cell ${ }_{55}$ is given by its age and the number of intracellular molecules, which evolve from birth to 56 division. After cell division, the mother's molecules are inherited by the two daughters ${ }_{57}$ through stochastic partitioning of molecules. Cell divisions occur asynchronously in the 58 population because cells divide at random times. In consequence, cell ages and molecule 59 numbers are heterogeneous in the population. Fig. 117 illustrates the resulting branching 60 process whose final state is a snapshot of the cell population. 
The seminal experiment by Elowitz et al. identified the sources of cell-to-cell variation 62 using snapshots of cells expressing green and red fluorescent reporters ${ }^{1}$. Reporters expressed ${ }_{63}$ at different levels appear either red or green, a signature of intrinsic noise. Cells with similar ${ }_{64}$ reporter levels appear yellow but with variable intensities, a signature of extrinsic noise. In 65 our model (Fig. 1a), similar effects are observed since stochasticity in biochemical reactions ${ }_{66}$ and partitioning of molecules at division account for intrinsic variation across the population. $\quad 67$ Cell age and variability in division timing provide a source of extrinsic noise (Fig. 1 b). In ${ }_{68}$ contrast to the population-view, the dynamics of isolated cells can also be tracked over time, 69 which we will refer to as lineage (Fig. 1 $\mathrm{c}$ ), which corresponds to a random path in the tree. 70 These statistics can differ significantly from population snapshots (Fig. 11d). To develop a ${ }_{71}$ quantitative understanding of these effects, we begin with deriving analytical framework to 72 quantify these populations.

\section{A Agent-based framework for stochastic biochemical kinetics in growing cell popula- 74 tions

To each cell we associate an age $\tau$ that counts the time since its last division and a number $\quad 76$ of set of biochemical species $X_{1}, X_{2}, \ldots, X_{N_{S}}$ present in amounts $x=\left(x_{1}, x_{2}, \ldots, x_{N_{S}}\right)$. ${ }_{77}$ These species interact via a network of $R$ intracellular biochemical reactions of the type $\quad 78$

$$
\nu_{1, r}^{+} X_{1}+\ldots+\nu_{N_{S}, r}^{+} X_{N_{S}} \rightarrow \nu_{1, r}^{-} X_{1}+\ldots+\nu_{N_{S}, r}^{-} X_{N_{S}}
$$

where $\nu_{i r}^{ \pm}$are the stoichiometric coefficients and $r=1, \ldots, R$. In the following, we outline the $\quad 79$ master equation that allows to analytically study these networks in an agent-based context. 80

\section{Master equation for the agent-based population}

The state of the overall cell population can be characterised by the snapshot density $\quad 82$ $n(\tau, x, t)$ that counts the number of cells at time $t$ with age between $\tau$ and $\tau+\mathrm{d} \tau$ and ${ }_{83}$ molecule counts $x$. Accordingly, the total number of cells in the population is given by 84

$$
N(t)=\int_{0}^{\infty} \mathrm{d} \tau \sum_{x} n(\tau, x, t),
$$

where the summation is over all possible molecule number configurations $x$. 
We assume that cells divide with an age-dependent rate $\gamma(\tau)$, which is related to the ${ }_{86}$ interdivision time distribution $\varphi\left(\tau_{d}\right)$ via

$$
\gamma(\tau)=\frac{\varphi(\tau)}{\int_{\tau}^{\infty} \mathrm{d} \tau_{d} \varphi\left(\tau_{d}\right)} .
$$

The snapshot density then evolves due to age-progression of cells, cell divisions and the 88 change in their molecular decomposition due to biochemical reactions

$$
\left(\frac{\partial}{\partial t}+\frac{\partial}{\partial \tau}+\gamma(\tau)\right) n(\tau, x, t)=\mathbb{Q} n(\tau, x, t) .
$$

Here, the change in the molecule numbers per cell is expressed by the transition matrix $\mathbb{Q} 90$ acting as $\mathbb{Q} n(\tau, x, t)=\sum_{r=1}^{R}\left[w_{r}\left(x-\nu_{r}\right) n\left(\tau, x-\nu_{r}, t\right)-w_{r}(x) n(\tau, x, t)\right]$, where $\left(\nu_{r}\right)_{i}=\nu_{i r}^{+}-\nu_{i r}^{-} \quad 91$ is the stochiometric vector of the $r^{t h}$ reaction. Cell birth is described by the boundary ${ }_{92}$ condition

$$
n(0, x, t)=2 \int_{0}^{\infty} \mathrm{d} \tau \sum_{x^{\prime}} B\left(x \mid x^{\prime}\right) \gamma(\tau) n\left(\tau, x^{\prime}, t\right),
$$

whereby the mother cell is replaced with two daughter cells of zero age with its molecules be- ${ }_{94}$ ing partitioned between them. The division kernel $B\left(x \mid x^{\prime}\right)$ is the probability of partitioning 95 the molecule numbers $x^{\prime}$ to $x$ of any daughter cell and is given by

$$
B\left(x \mid x^{\prime}\right)=\frac{1}{2} B_{1}\left(x \mid x^{\prime}\right)+\frac{1}{2} B_{2}\left(x \mid x^{\prime}\right),
$$

where $B_{1}$ and $B_{2}$ are the marginal probabilities for the two daughter cells to inherit $x$ of $\quad{ }_{97}$ the mother cell's molecules $x^{\prime}$. Importantly, if the total amount is conserved in the division, 98 we have $B_{2}\left(x \mid x^{\prime}\right)=B_{1}\left(x^{\prime}-x \mid x^{\prime}\right)$. Thus each cell inherits an equal amount of molecules ${ }_{99}$ $E_{B}\left[x \mid x^{\prime}\right]=x^{\prime} / 2$, because we do not distinguish the daughters. 100

Since resolving the time-evolution of the snapshot density is a formidable task, we con- 101 centrate on the long-term evolution of Eq. (3), which describes the exponential growth phase 102 or balanced growth condition. In this limit, the total number of cells grows exponentially 103 $N(t) \sim N_{0} e^{\lambda t}$ with rate $\lambda$ and the fraction of cells with a certain cell age and molecule ${ }_{104}$ content is constant

$$
\Pi(\tau, x)=\lim _{t \rightarrow \infty} \frac{n(\tau, x, t)}{N(t)},
$$

due to the balance between cell births, divisions and the biochemical reactions. In the 106 following, we summarise how to characterise this distribution analytically. 
The fraction of cells with the same age in a snapshot is given by the age-distribution, 109 which follows 31

$$
\Pi(\tau)=\lim _{t \rightarrow \infty} \sum_{x} \frac{n(\tau, x, t)}{N(t)}=2 \lambda e^{-\lambda \tau} \int_{\tau}^{\infty} \mathrm{d} \tau^{\prime} \varphi\left(\tau^{\prime}\right) .
$$

The distribution $\varphi$ characterises the interdivision times

$$
\varphi\left(\tau_{d}\right)=\gamma\left(\tau_{d}\right) e^{-\int_{0}^{\tau_{d}} \mathrm{~d} \tau^{\prime} \gamma\left(\tau^{\prime}\right)}
$$

as also seen from Eq. (2). The age distribution, Eq. (6), depends on the population growth 112 rate $\lambda$ that is uniquely determined by the Euler-Lotka equation

$$
1=2 \int_{0}^{\infty} \mathrm{d} \tau_{d} e^{-\lambda \tau} \varphi\left(\tau_{d}\right)
$$

The above equations constitute the fundamental age-structure of microbial populations, 114 which has been verified in experiments 16132133 .

We consider the total number of cells with age $\tau$ and molecule count $x$ divided by the ${ }_{117}$ number of cells at that age. This conditional probability quantifies the likelihood of observing 118 $x$ molecules in a cell of age $\tau$ and is given by

$$
\Pi(x \mid \tau)=\lim _{t \rightarrow \infty} \frac{n(\tau, x, t)}{\sum_{x} n(\tau, x, t)}=\frac{\Pi(\tau, x)}{\Pi(\tau)} .
$$

It can be verified ${ }^{31}$ that $\Pi(x \mid \tau)$ obeys

$$
\frac{\partial}{\partial \tau} \Pi(x \mid \tau)=\mathbb{Q} \Pi(x \mid \tau)
$$

which is similar to the chemical master equation (with time replaced by cell age). An ${ }_{121}$ important difference though, is that it has to be solved subject to the boundary condition $\quad 122$

$$
\Pi(x \mid 0)=\int_{0}^{\infty} \mathrm{d} \tau_{d} \sum_{x^{\prime}} B\left(x \mid x^{\prime}\right) \Pi\left(x^{\prime} \mid \tau_{d}\right) \rho\left(\tau_{d}\right),
$$

which accounts for the cell divisions. The distribution under the integral

$$
\rho\left(\tau_{d}\right)=2 \varphi\left(\tau_{d}\right) e^{-\lambda \tau_{d}}
$$


is the interdivision time distribution in the population ${ }^{32 \mid 34}$. The distribution describes the ${ }_{124}$ interdivision times of cells with completed cell divisions and depends explicitly on the pop- ${ }_{125}$ ulation growth rate $\lambda$.

A lineage tracks a single of the population over successive cell divisions. In the long term 128 its evolution also approaches a stable distribution, which we denote by $\pi(\tau, x)$. The molecule 129 number distribution for cells of the same age in a lineage is given by $\pi(x \mid \tau)=\pi(x, \tau) / \pi(\tau) \quad{ }_{130}$ and satisfies

$$
\begin{aligned}
& \frac{\partial}{\partial \tau} \pi(x \mid \tau)=\mathbb{Q} \pi(x \mid \tau) \\
& \pi(x \mid 0)=\int_{0}^{\infty} \mathrm{d} \tau_{d} \sum_{x^{\prime}} B\left(x \mid x^{\prime}\right) \Pi\left(x^{\prime} \mid \tau_{d}\right) \varphi\left(\tau_{d}\right)
\end{aligned}
$$

By comparing the above equations with Eqs. (10), we notice that this distribution is obtained 132 by substituting the division time distribution $\rho$ by $\varphi$. Thus cells of the same age can be ${ }_{133}$ analysed using a unified framework whether in populations or lineages. The age-distribution 134 in a lineage, however, which is

$$
\pi(\tau)=\frac{1}{E_{\varphi}[\tau]} \int_{\tau}^{\infty} \mathrm{d} \tau^{\prime} \varphi\left(\tau^{\prime}\right)
$$

differs significantly from the population, Eq. (6).

In many practical situations, solving for the full distribution is infeasible. Summary 138 statistics such as means and variances, which we will focus on in the following, present 139 convenient alternatives as they are more amenable to analysis. 
In brief, the moment equations are obtained by multiplying Eq. 10a by $x$ or $x x^{T}$ and ${ }_{142}$ summing over all possible states. The results for the first and second moments are

$$
\begin{aligned}
& \frac{\partial E_{\Pi}[x \mid \tau]}{\partial \tau}= \sum_{r=1}^{R} \nu_{r} E_{\Pi}\left[w_{r}(x) \mid \tau\right] \\
& \frac{\partial E_{\Pi}\left[x x^{T} \mid \tau\right]}{\partial \tau}=\sum_{r=1}^{R}\left(\nu_{r} E_{\Pi}\left[x^{T} w_{r}(x) \mid \tau\right]+E_{\Pi}\left[x w_{r}(x) \mid \tau\right] \nu_{r}^{T}\right) \\
&+\sum_{r=1}^{R}\left(\nu_{r} E_{\Pi}\left[w_{r}(x) \mid \tau\right] \nu_{r}^{T}\right) .
\end{aligned}
$$

Interestingly, these are the same moment equations that appear in the study of systems 144 without age-dependence (with age being replaced by the observational time). The key ${ }_{145}$ difference is the boundary condition subject to which the moment equations have to be 146 solved. These conditions follow from Eq. (10b) and the conservation of molecules in Eq. (4), 147 which implies $E_{B}\left[x \mid x^{\prime}\right]=x^{\prime} / 2$. They read

$$
\begin{aligned}
& E_{\Pi}[x \mid 0]=\frac{1}{2} E_{\rho}\left[E_{\Pi}\left[x \mid \tau_{d}\right]\right], \\
& E_{\Pi}\left[x x^{T} \mid 0\right]=E_{\rho}\left[E_{\Pi}\left(E_{B}\left[x^{\prime} x^{\prime T} \mid x\right] \mid \tau_{d}\right)\right] .
\end{aligned}
$$

The first condition states that, on average, molecule numbers need to double over one cell ${ }_{149}$ cycle. The second condition relates the second moments to the partitioning of molecules 150 described by the division kernel, Eq. 4 .

We now consider the snapshot moments of molecule numbers irrespective of age. $E_{\Pi}[x]={ }_{153}$ $E_{\Pi}\left[E_{\Pi}[x \mid \tau]\right]$ and $E_{\Pi}\left[x x^{T}\right]=E_{\Pi}\left[E_{\Pi}\left[x x^{T} \mid \tau\right]\right]$ are obtained by multiplying Eq. (14) with $\Pi(\tau) \quad 154$ and performing the integration. For this purpose, consider the expected value of a function 155 $f(\tau)$ with respect to the age distribution, which satisfies

$$
\begin{aligned}
\int_{0}^{\infty} \mathrm{d} \tau & \Pi(\tau) \frac{\partial f(\tau)}{\partial \tau} \\
& =-\int_{0}^{\infty} \mathrm{d} \tau f(\tau) \frac{\partial}{\partial \tau} \Pi(\tau)-f(0) \Pi(0) \\
& =\lambda E_{\Pi}[f(\tau)]+\lambda E_{\rho}(f(\tau)-2 f(0)) .
\end{aligned}
$$


In the first line, we integrate by parts assuming $\lim _{\tau \rightarrow \infty} f(\tau) \Pi(\tau)=0$, and in the second ${ }_{157}$ line we substituted Eq. (6) for $\Pi(\tau)$ and performed the derivative. The first term captures 158 the effect of dilution, while the second term describes discrete changes during cell division. $\quad 159$

Setting now $f(\tau)=E_{\Pi}[x \mid \tau]$ in (15) and combing the result with Eq. (14a) and the 160 boundary conditions $14 \mathrm{c}$, we find an equation for the mean number of molecules in the ${ }_{161}$ population,

$$
\lambda E_{\Pi}[x]=\sum_{r=1}^{R} \nu_{r} E_{\Pi}\left[w_{r}(x)\right] .
$$

Similarly, using Eq. 15 with $f(\tau)=E_{\Pi}\left[x x^{T} \mid \tau\right]$, Eq. 14b and $114 \mathrm{~d}$, the equation for the ${ }_{163}$ second moment becomes

$$
\begin{aligned}
\lambda E_{\Pi}\left[x x^{T}\right]+ & \left.\frac{\lambda}{2} E_{\rho}\left[E_{\Pi}\left(x x^{T} \mid \tau_{d}\right)\right]-4 E_{\Pi}\left(\operatorname{Cov}_{B}\left[x^{\prime} \mid x\right] \mid \tau\right)\right] \\
= & \sum_{r=1}^{R}\left(\nu_{r} E_{\Pi}\left[x^{T} w_{r}(x)\right]+E_{\Pi}\left[x w_{r}(x)\right] \nu_{r}^{T}\right) \\
& +\sum_{r=1}^{R} \nu_{r} E_{\Pi}\left[w_{r}(x)\right] \nu_{r}^{T},
\end{aligned}
$$

where the left hand side depends explicitly on the division-time distribution $\rho$. Obviously, 165 these equations cannot be solved in general, not only because the hierarchy of moments is 166 not closed but also because they depend on moments for cells of known age. The conditions ${ }_{167}$ for which these equations are closed and can be solved exactly are discussed in SI V C, $\quad{ }_{168}$

\section{Results}

\section{A Decomposing noise into intrinsic and extrinsic contributions}

To circumvent the moment-closure problem, we employ the linear noise approximation 171

to decompose the noise into intrinsic and extrinsic components ( $\sec ^{\sqrt{35 \mid 36}}$ for details of the 172 approximation). In brief, the approximation assumes Gaussian fluctuations and provides 173 closed-form expressions for the mean molecule numbers and their covariances. Writing short ${ }_{174}$ $\operatorname{Cov}_{\Pi}[x \mid \tau]=E_{\Pi}\left[x x^{T} \mid \tau\right]-E_{\Pi}[x \mid \tau] E_{\Pi}\left[x^{T} \mid \tau\right]$ for the covariance matrix, the result is

$$
\begin{aligned}
\frac{\partial}{\partial \tau} E_{\Pi}[x \mid \tau] & =\nu w\left(E_{\Pi}[x \mid \tau]\right), \\
\frac{\partial}{\partial \tau} \operatorname{Cov}_{\Pi}[x \mid \tau] & =\mathcal{J} \operatorname{Cov}_{\Pi}[x \mid \tau]+\operatorname{Cov}_{\Pi}[x \mid \tau] \mathcal{J}^{T}+\mathcal{D} .
\end{aligned}
$$


where the Jacobian $\mathcal{J}$ and the diffusion matrix $\mathcal{D}$ are defined as

$$
\begin{aligned}
\mathcal{J}_{i j}(\tau) & =\sum_{r=1}^{R} \nu_{i r} \frac{\partial w_{r}\left(E_{\Pi}[x \mid \tau]\right)}{\partial E_{\Pi}\left[x_{j} \mid \tau\right]}, \\
\mathcal{D}_{i j}(\tau) & =\sum_{r=1}^{R} \nu_{i r} \nu_{j r} w_{r}\left(E_{\Pi}[x \mid \tau]\right),
\end{aligned}
$$

which depend on cell age through the mean molecule numbers $E_{\Pi}[x \mid \tau]$. Comparison of 177 Eqs. (18) with (14) shows that these equations are exact whenever the propensities are 178 linear in the molecule numbers. In all other cases, we consider these as an approximation 179 valid in the limit of large molecule numbers.

Next, we cast the boundary condition $(14 \mathrm{~d}]$ in terms of the covariance matrix $\operatorname{Cov}_{\Pi}[x \mid \tau], \quad 181$ which leads to

$$
\begin{aligned}
E_{\Pi}[x \mid 0]= & \frac{1}{2} E_{\rho}\left[E_{\Pi}\left(x \mid \tau_{d}\right)\right] \\
\operatorname{Cov}_{\Pi}\left[x_{i}, x_{j} \mid 0\right]= & \underbrace{\frac{1}{4} E_{\rho}\left[\operatorname{Cov}_{\Pi}\left(x_{i}, x_{j} \mid \tau\right)\right]}_{\text {variability between mother cells }} \\
& +\underbrace{E_{\rho}\left[E_{\Pi}\left(\operatorname{Cov}_{B}\left[x_{i}, x_{j} \mid x^{\prime}\right] \mid \tau\right)\right]}_{\text {partitioning of molecules }} \\
& +\underbrace{\frac{1}{4} \operatorname{Cov}_{\rho}\left[E_{\Pi}\left(x_{j} \mid \tau\right), E_{\Pi}\left(x_{j} \mid \tau\right)\right]}_{\text {cell cycle variability }}
\end{aligned}
$$

The first term is the contribution due to fluctuations in the number of molecules before ${ }_{183}$ division. The second term denotes the variation due to random partitioning of molecules at 184 cell division, while the third contribution stems from differences in the molecule numbers 185 due to different cell cycle lengths. We note that Eqs. 20b themselves do not constitute a ${ }_{186}$ noise decomposition since these contribute do not propagate independently. Instead, they ${ }_{187}$ represent the sources of cell-to-cell variability for the two daughter cells.

To investigate how the different sources of variations affect biochemical reaction dynamics, 190 we consider the synthesis and degradation of mRNA molecules and translation into proteins 191

$$
\begin{aligned}
& \varnothing \stackrel{k_{0}}{\longrightarrow} \text { mRNA } \stackrel{k_{m}}{\longrightarrow} \varnothing, \\
& \text { mRNA } \stackrel{k_{s}}{\longrightarrow} \text { mRNA + Protein. }
\end{aligned}
$$


We do not account for protein degradation explicitly in this model since stable proteins are 192 diluted in the population by cell division, the effect of which we will study in the following. 193 For simplicity, we assume that mRNA degradation is faster than the population growth 194 such that the reactions can be approximated by a single reaction synthesising proteins in 195 stochastic bursts. At the same time, for the purpose of the noise decomposition, we consider 196 an additional, identical copy of the same circuit in the cell

$$
\begin{aligned}
& \varnothing \stackrel{k_{0}}{\rightarrow} m_{1} \times \text { Protein }_{1}, \\
& \varnothing \stackrel{k_{0}}{\rightarrow} m_{2} \times \text { Protein }_{2} .
\end{aligned}
$$

The stochastic burst size of the first and second copy are denoted by $m_{1}$ and $m_{2}$, respectively, 198 and both follow a geometric distribution with mean $b=k_{s} / k_{m}$ (see Ref. $\stackrel{37}{ }$ and SI VD for ${ }_{199}$ details of the burst approximation). 200

a. Mean number of proteins.

Since the two reporter proteins are expressed identically in the cell, their mean expression 202 levels must be the same. Denoting the protein numbers of the two reporters by $x_{1}$ and $x_{2},{ }_{203}$ we have $E_{\Pi}\left[x_{1} \mid \tau\right]=E_{\Pi}\left[x_{2} \mid \tau\right]$. The rate equation (18a) for the average number of proteins ${ }_{204}$ for a cell of given age becomes

$$
\frac{\partial E_{\Pi}\left[x_{1} \mid \tau\right]}{\partial \tau}=k_{0} b
$$

The solution that respects the boundary condition 20a is

$$
E_{\Pi}\left[x_{1} \mid \tau\right]=k_{0} b\left(\tau+E_{\rho}[\tau]\right) .
$$

The number of proteins inherited after cell division $(\tau=0)$ is thus $k_{0} b E_{\rho}[\tau]$, which depends ${ }_{207}$ on the mean division time $E_{\rho}[\tau]$ in the population.

b. Separating noise into intrinsic and extrinsic components.

For identical two-reporter systems, the overall variance can be decomposed into intrinsic 210 and extrinsic components as follows

$$
\Sigma(\tau)=\Sigma_{\text {int }}(\tau)+\Sigma_{\text {ext }}(\tau)
$$

The individual contributions can be quantified using ${ }^{1}$

$$
\begin{aligned}
\Sigma_{\text {int }}(\tau) & =\frac{1}{2} E_{\Pi}\left[\left(x_{1}-x_{2}\right)^{2} \mid \tau\right], \\
\Sigma_{\text {ext }}(\tau) & =\operatorname{Cov}_{\Pi}\left[x_{1}, x_{2} \mid \tau\right] .
\end{aligned}
$$


Since these components are measured in the same cell, they also account for the correct ${ }_{213}$ history dependence $\mathrm{C}^{7 / 9138}$.

The variance of intrinsic and extrinsic fluctuations follows from using Eqs. (25) in (18b) 215 and rearranging, which leads to

$$
\frac{\partial}{\partial \tau} \Sigma_{\text {int }}(\tau)=b(2 b+1) k_{0}, \quad \frac{\partial}{\partial \tau} \Sigma_{\text {ext }}(\tau)=0 .
$$

Its solution is obtained by straight-forward integration and is given by $\Sigma_{\text {int }}(\tau)=\Sigma_{\text {int }}(0)+{ }^{217}$ $b(2 b+1) k_{0} \tau$ and $\Sigma_{\text {ext }}(\tau)=\Sigma_{\text {ext }}(0)$. To fix the boundary condition (20b), we assume that ${ }_{218}$ each molecule of the mother cell being partitioned with equal probability between the two ${ }_{219}$ daughter cells. In this case, the division kernel in Eq. (4) is binomial with covariance 220 $\operatorname{Cov}_{B}\left[x_{i}, x_{j} \mid x^{\prime}\right]=\delta_{i j} x_{i}^{\prime} / 4$. We then find that $\Sigma_{\text {int }}(0)=\frac{1}{3} b(2 b+3) k_{0} E_{\rho}[\tau]$ and $\Sigma_{\text {ext }}(0)={ }^{221}$ $\frac{1}{3} b^{2} k_{0}^{2} E_{\rho}[\tau]^{2} \mathrm{CV}_{\rho}^{2}$, and finally

$$
\begin{aligned}
& \mathrm{CV}_{\text {int }}^{2}(\tau)=\frac{\Sigma_{\text {int }}(\tau)}{E_{\Pi}\left[x_{1} \mid \tau\right]^{2}}=\frac{1}{E_{\Pi}\left[p_{1} \mid \tau\right]}\left(1+\frac{2}{3} b\left(1+\frac{2 \tau}{E_{\rho}[\tau]+\tau}\right)\right), \\
& \mathrm{CV}_{\text {ext }}^{2}(\tau)=\frac{\Sigma_{\text {ext }}(\tau)}{E_{\Pi}\left[x_{1} \mid \tau\right]^{2}}=\frac{E_{\rho}[\tau]^{2}}{\left(E_{\rho}[\tau]+\tau\right)^{2}} \frac{\mathrm{CV}_{\rho}^{2}}{3}
\end{aligned}
$$

The coefficients of variations quantify the size of fluctuations relative to the mean. The result ${ }^{223}$ confirms the intuition that intrinsic noise decreases with the mean number of molecules. The 224 extrinsic noise component, however, reflects the variations in cell cycle duration $\mathrm{CV}_{\rho}^{2}$ that ${ }_{225}$ are transmitted to the protein levels.

c. Snapshots display higher intrinsic but lower extrinsic noise levels than lineages. 227

Next, we compare the statistics of snapshots of a growing population with the one of ${ }_{228}$ a lineage of an isolated cell over time. As explained in Sec. II A 4, we obtain the lineage ${ }_{229}$ statistic by substituting the division time distribution $\rho$ for $\varphi$ in Eqs. (23), 26a) and (26b). 230

Interestingly, the deviations between these two statistics is apparent even on the mean 231 level. To see this, we notice that the mean number of molecules, Eq. (23), increases with the ${ }_{232}$ duration of the cell cycle. It is well known that the cell cycle time is longer when averaged ${ }_{233}$ over single cells than for cells in the population ${ }^{16} E_{\rho}[\tau] \leq E_{\varphi}[\tau]$. An intuitive explanation of ${ }^{234}$ this fact is that fast dividing cells are over-represented in the population. It hence follows ${ }_{235}$ from Eq. 23) that the expected number of molecules is lower in populations compared to ${ }_{236}$ lineages, no matter what the division time distribution is.

In Fig. 2a, we compare the total noise $\mathrm{CV}_{\text {tot }}^{2}(\tau)=\mathrm{CV}_{\text {int }}^{2}(\tau)+\mathrm{CV}_{\text {ext }}^{2}(\tau)$ for gamma and ${ }_{238}$ log-normal distributed interdivision times. In both cases, we observe that the noise exhibits 239 
a maximum for low cell cycle variability. With increasing cell cycle variability, we find that 240 the maximum flattens in the lineage but not in snapshot statistics. Albeit the two statistics 241 are collected from different samples of the same population, snapshots are more noisy than 242 lineages in both cases. To understand this noise propagation, we decompose the total noise 243 into intrinsic and extrinsic components via Eqs. (26a) and 26b). We observe that intrinsic ${ }_{244}$ noise in snapshots increases with cell cycle variability (Fig. 2b) while it is significantly lower ${ }_{245}$ in lineages and independent of these fluctuations, which is consistent with lower expression 246 levels in snapshots.

Fig. 2 a also reveals a non-monotonic dependence of the intrinsic noise component on 248 cell age. To explain this phenomenon, we notice that intrinsic noise, Eq. 26a), increases ${ }^{249}$ with cell age due to an increase in the Fano factor. For older cells, however, intrinsic noise ${ }_{250}$ decreases with age as these cells express higher protein levels. Combining these findings 251 explains the noise maximum at a well-defined cell age. By maximising Eq. (26a) over all ${ }^{252}$ possible cell ages, the age at which noise peaks is $\tau / E_{\rho}\left[\tau_{d}\right]=\frac{2 b-3}{6 b+3}$ whenever $b>3 / 2$. This ${ }^{253}$ ratio only depends on the burst size $b$ and approaches $1 / 3$ of the mean cell cycle time for ${ }_{254}$ large $b$. By contrast, we find that extrinsic noise is lower and decays slower over the cell cycle 255 in snapshots than in lineages (Fig. 2e). We conclude that lineage statistics may significantly 256 underestimate intrinsic heterogeneity but overestimate extrinsic noise in the population. In 257 the next subsection, we extend this method to general stochastic reaction networks.

\section{General decomposition for cells of the same age}

We now generalise the decomposition to two-reporter systems involving an arbitrary 260 network of biochemical reactions. As before, we assume that the two copies of our network 261 with molecule numbers $x_{1}$ and $x_{2}$ do not interact and involve the same type reactions, and ${ }_{262}$ therefore they have the same mean expression level. The covariance of the two-reporter ${ }_{263}$ system is

$$
\operatorname{Cov}_{\Pi}\left[x_{1}, x_{2} \mid \tau\right]=\left(\begin{array}{cc}
\Sigma_{11}(\tau) & \Sigma_{12}(\tau) \\
\Sigma_{12}(\tau) & \Sigma_{11}(\tau)
\end{array}\right)
$$


whose individual components satisfy

$$
\begin{aligned}
\frac{\partial}{\partial \tau} \Sigma_{11}(\tau) & =\mathcal{J} \Sigma_{11}+\Sigma_{11} \mathcal{J}^{T}+\mathcal{D} \\
\frac{\partial}{\partial \tau} \Sigma_{12}(\tau) & =\mathcal{J} \Sigma_{12}+\Sigma_{12} \mathcal{J}^{T},
\end{aligned}
$$

according to Eqs. $18 \mathrm{~b}$.

The intrinsic and extrinsic noise components can be expressed via

$$
\begin{aligned}
& \Sigma_{\text {int }}(\tau)=\frac{1}{2} E\left[\left(x_{1}-x_{2}\right)^{2} \mid \tau\right]=\Sigma_{11}-\Sigma_{12}, \\
& \Sigma_{\text {ext }}(\tau)=\operatorname{Cov}\left[x_{1}, x_{2} \mid \tau\right]=\Sigma_{12} .
\end{aligned}
$$

Since the covariances obey the linear equations (27), the two noise contributions evolve 268 independently. In particular, the intrinsic and extrinsic covariances satisfy

$$
\begin{aligned}
\frac{\mathrm{d}}{\mathrm{d} \tau} \Sigma_{\text {int }} & =\mathcal{J} \Sigma_{\text {int }}+\Sigma_{\text {int }} \mathcal{J}^{T}+\mathcal{D}, \\
\frac{\mathrm{d}}{\mathrm{d} \tau} \Sigma_{\text {ext }} & =\mathcal{J} \Sigma_{\text {ext }}+\Sigma_{\text {ext }} \mathcal{J}^{T}
\end{aligned}
$$

where only the intrinsic component involves the biochemical noise from the intracellular 270 reactions through the diffusion matrix $\mathcal{D}$.

Assuming again binomial partitioning with covariance $\operatorname{Cov}_{B}\left[x_{i}, x_{j} \mid x^{\prime}\right]=\delta_{i j} x_{i}^{\prime} / 4$, allows us 272 to split the boundary condition (20b) according to Eq. (24). The results are two independent ${ }_{273}$ conditions

$$
\begin{aligned}
4 \Sigma_{\text {int }}(0)=E_{\rho}[ & \left.\Sigma_{\text {int }}\left(\tau_{d}\right)\right]+E_{\rho}\left[E_{\Pi}\left[\operatorname{diag}(x) \mid \tau_{d}\right]\right], \\
4 \Sigma_{\text {ext }}(0)=E_{\rho}[ & {\left[\Sigma_{\text {ext }}\left(\tau_{d}\right)\right] } \\
& \quad+\operatorname{Cov}_{\rho}\left[E_{\Pi}\left[x \mid \tau_{d}\right], E_{\Pi}\left[x \mid \tau_{d}\right]\right] .
\end{aligned}
$$

The noise decomposition is fully specified by the mean number of molecules for cells of the ${ }_{275}$ same age, the Jacobian $\mathcal{J}$ of the corresponding rate equations, the diffusion matrix $\mathcal{D}$ (see ${ }_{276}$ Eqs. (19)) and the distribution of interdivision times in the population $\rho$ (see Eq. (11)). 277 Importantly, Eq. 28c shows that partitioning is a noise source to intrinsic fluctuations, 278 while Eq. (28d) shows that cell cycle variations contribute to extrinsic fluctuations. We 279 conclude that conditioning on the cell cycle position is not enough to eliminate all extrinsic 280 noise. Before we continue, we note that other types of partitioning, such as asymmetric ${ }_{281}$ cell division, can be easily incorporated into the framework but using a different form of ${ }_{282}$ $\operatorname{Cov}_{B}\left[x_{i}, x_{j} \mid x^{\prime}\right]^{39}$. 
An obstacle for applying this decomposition in practice is that in many situation the cell 285 age is not known, and this is especially true for population snapshots. For this reason, the 286 mean of the molecule number has to be averaged over all possible cell ages

$$
E_{\Pi}[x]=E_{\Pi}\left(E_{\Pi}[x \mid \tau]\right)=\int_{0}^{\infty} \mathrm{d} \tau E_{\Pi}[x \mid \tau] \Pi(\tau) .
$$

Similarly, we use the law of total variance to decompose the snapshot-variance as

$$
\bar{\Sigma}=\underbrace{\bar{\Sigma}_{\text {int }}}_{\text {intrinsic }}+\underbrace{\bar{\Sigma}_{\mathrm{cc}}+\bar{\Sigma}_{\text {age }}}_{\text {extrinsic }}
$$

with

$$
\begin{aligned}
& \bar{\Sigma}_{\text {int }}=E_{\Pi}\left(\Sigma_{\text {int }}(\tau)\right), \\
& \bar{\Sigma}_{\text {cc }}=E_{\Pi}\left(\Sigma_{\text {ext }}(\tau)\right), \\
& \bar{\Sigma}_{\text {age }}=\operatorname{Cov}_{\Pi}\left(E_{\Pi}[x \mid \tau], E_{\Pi}[x \mid \tau]\right) .
\end{aligned}
$$

The first term in Eq. 29a is the intrinsic variance measured across a population, the second 290 term is the extrinsic variance transmitted from cell cycle variations, and the third term is 291 another extrinsic component that comes from averaging over cells of different ages. The total ${ }_{292}$ extrinsic noise, which is measured in a two-reporter system, is the sum of the second and 293 third term. The practical use of this noise decomposition is demonstrated in the following 294 section.

\section{B Practical computation of the noise decomposition and applications}

Finally, we apply the noise decomposition to analyse snapshots in which the age of in- ${ }^{297}$ dividual cells is not known. While the decomposition can be carried out exactly for linear 298 reaction networks, we also outline a numerical method with which the decomposition can be ${ }^{299}$ carried out efficiently for complex nonlinear networks as we demonstrate for a protein that 300 regulates its own expression. 
For linear reaction networks in which the propensities are linear functions of the molecule 303 numbers. This dependence allows to average the statistics exactly over all cell ages. Thus 304 from Eq. (16), we obtain the rate equations

$$
\lambda E_{\Pi}[x]=\nu w\left(E_{\Pi}[x]\right),
$$

since $E_{\Pi}[w(x)]=w\left(E_{\Pi}[x]\right)$. These equations coincide precisely with the steady state of the 306 traditional deterministic rate equations including an effective dilution term proportional to 307 the population growth rate $\lambda$.

Averaging Eq. 28a) over all ages and accounting for the boundary terms using Eq. (15), 309 the intrinsic variance becomes

$$
\begin{aligned}
\lambda \bar{\Sigma}_{\mathrm{int}}= & \mathcal{J} \bar{\Sigma}_{\mathrm{int}}+\bar{\Sigma}_{\mathrm{int}} \mathcal{J}^{T}+E_{\Pi}[\mathcal{D}(\tau)] \\
& +\lambda\left(2 \Sigma_{\mathrm{int}}(0)-E_{\rho}\left[\Sigma_{\mathrm{int}}\left(\tau_{d}\right)\right]\right)
\end{aligned}
$$

where the Jacobian $\mathcal{J}$ is assumed to be independent of cell age. Similarly, averaging 311 Eq. (28b) the extrinsic variance transmitted from cell cycle fluctuations is obtained as

$$
\begin{aligned}
\lambda \bar{\Sigma}_{\mathrm{cc}}= & \mathcal{J} \bar{\Sigma}_{\mathrm{cc}}+\bar{\Sigma}_{\mathrm{cc}} \mathcal{J}^{T} \\
& +\lambda\left(2 \Sigma_{\text {ext }}(0)-E_{\rho}\left[\Sigma_{\text {ext }}\left(\tau_{d}\right)\right]\right) .
\end{aligned}
$$

Similarly, an equation for $\bar{\Sigma}_{\text {age }}$ can be derived (see SI VB for details), which reads

$$
\begin{aligned}
\lambda \bar{\Sigma}_{\text {age }}= & \mathcal{J} \bar{\Sigma}_{\text {age }}+\bar{\Sigma}_{\text {age }} \mathcal{J}^{T} \\
& +\lambda\left(E_{\Pi}[x] E_{\Pi}\left[x^{T}\right]-2 E_{\Pi}[x \mid 0] E_{\Pi}\left[x^{T} \mid 0\right]\right. \\
& \left.\quad-\operatorname{Cov}_{\rho}\left(E_{\Pi}\left[x \mid \tau_{d}\right], E_{\Pi}\left[x \mid \tau_{d}\right]\right)\right)
\end{aligned}
$$

This decomposition exactly characterises the variability of linear intracellular reaction net- ${ }_{314}$ works across snapshots.

a. Application to stochastic reporter expression.

We return to the two-reporter system $(22)$ and apply the decomposition developed in the 317 previous section. From Eq. (30), we find that the mean molecule number is given by

$$
E[p]= \begin{cases}k_{0} b / \lambda & \text { snapshot } \\ b k_{0}\left(E_{\varphi}\left[\tau_{d}\right]+E_{\pi}[\tau]\right) & \text { lineage. }\end{cases}
$$


Note that the lineage mean follows from integrating Eq. 23) with $\varphi$ instead of $\rho$ against 319 the lineage age-distribution (13). Interestingly, only the population mean agrees with the 320 intuition in which the ratio of synthesis and dilution rates yields the steady state levels. 321 However, both averages depend implicitly on the cell cycle variability through the average 322 age $E_{\pi}[\tau]$ or the population growth rate $\lambda$, respectively. In Fig. 3 a, we show that molecule 323 numbers in the lineage increase with cell cycle variability while they decrease in the snapshot 324 statistic under the same conditions. These quantities thus exhibit opposite sensitivities to 325 cell cycle variability.

Next, we explore the noise properties of the reporter system using the decomposition 327 (31). We find that the contributions of intrinsic noise are

$$
\mathrm{CV}_{\text {int }}^{2}= \begin{cases}\frac{1}{E_{\Pi}[p]}\left(1+\frac{2}{3} b\left(1+2 \lambda E_{\Pi}[\tau]\right)\right) & \text { snapshot } \\ \frac{1}{E_{\pi}[p]}\left(1+2 b-\frac{4 b E_{\varphi}\left[\tau_{d}\right]}{3\left(E_{\varphi}\left[\tau_{d}\right]+E_{\pi}[\tau]\right)}\right) & \text { lineage }\end{cases}
$$

which is inversely proportional to the mean number of proteins. The contribution of extrinsic 329 noise due to stochasticity in cell cycle duration is

$$
\mathrm{CV}_{\mathrm{cc}}^{2}= \begin{cases}\frac{1}{3}\left(E_{\rho}\left[\tau_{d}\right] \lambda\right)^{2} \mathrm{CV}_{\rho}^{2}\left[\tau_{d}\right] & \text { snapshot } \\ \frac{1}{3} \frac{E_{\varphi}\left[\tau_{d}\right]^{2}}{\left(E_{\varphi}\left[\tau_{d}\right]+E_{\pi}[\tau]\right)^{2}} \mathrm{CV}_{\varphi}^{2}\left[\tau_{d}\right] & \text { lineage }\end{cases}
$$

and the one due to the unknown cell age is

$$
\mathrm{CV}_{\text {age }}^{2}= \begin{cases}\left(\lambda E_{\Pi}[\tau]\right)^{2} \mathrm{CV}_{\Pi}^{2}[\tau] & \text { snapshot } \\ \frac{E_{\pi}[\tau]^{2}}{\left(E_{\varphi}\left[\tau_{d}\right]+E_{\pi}[\tau]\right)^{2}} \mathrm{CV}_{\pi}^{2}[\tau] & \text { lineage. }\end{cases}
$$

The noise decomposition crucially depends on the population growth rate $\lambda$, while in lineages 332 it depends on the corresponding average cell age $E_{\pi}[\tau]$. More specifically, $\mathrm{CV}_{\mathrm{cc}}^{2}$ and $\mathrm{CV}_{\text {age }}^{2}{ }^{33}$ depend the variations in the age and interdivision time distributions, which are generally 334 different in lineages and population. We illustrate this dependence using the analytical 335 decompositions for two different interdivision-time distributions with the same mean and 336 variance.

For both the gamma and the log-normal distribution, intrinsic noise (red lines, Fig. 3b) 338 exhibits opposite sensitivities on cell cycle variability comparing lineage (dashed) and snap- ${ }_{339}$ shot statistics (solid). This observation is explained by smaller mean expression levels in 340 snapshots (cf. Fig. 3a) because intrinsic noise is expected to scale inversely with the mean 341 
molecule number. For the gamma distribution, the extrinsic noise transmitted from cell 342 cycle variations (blue lines, Fig. 3b) is (almost) identical for these measures. For the log- 343 normal distribution, however, extrinsic noise in the lineage is smaller than in the snapshot. 344 Interestingly, we find that the total noise is higher in snapshots than in lineages for the 345 gamma distribution (black lines, Fig. 35), while this not true for the log-normal distribution 346 and large cell cycle variability (black lines, Fig. 3d). 347

In developed network models, the extrinsic components will also depend on the biochem- 348 ical properties of the network. We demonstrate this analytically in SIVD when the protein 349 is also subject to degradation, which reveals intricate noise patterns. A straight-forward ap- 350 proach for the noise decomposition in complex biochemical network is given in the following 351 section.

\section{Decomposition for nonlinear reaction networks}

For nonlinear reaction networks, it is generally difficult to carry out the noise decomposi- 354 tion analytically. This is because the statistics of known and unkown cell age are intricately 355 coupled and can be solved simultaneously only in simple cases. An efficient and generally 356 applicable procedure to compute the numerical noise decomposition is the following: 357

1. Calculate the population growth rate using Eq. (8).

2. Solve for the statistics of cells of the same age, Eq. (28a) and (28b) and use the shooting 359 method to match the boundary conditions (28c) and (28d).

3. Obtain the noise decomposition 29a irrespectively of cell age by performing the 361 average in Eqs. (29b).

Step 1 can be efficiently computed using numerical root-finding methods. The shooting 363 method in Step 2 consists of an iterative procedure by which the mean molecule number, 364 intrinsic variance and extrinsic variance are obtained through an initial guess on their values 365 after cell division, $E_{\rho}[x \mid 0], \Sigma_{\text {int }}(0)$ and $\Sigma_{\mathrm{cc}}(0)$, and the result is then refined using standard 366 root-finding methods until the boundary conditions (28c) and (28d) are matched. Step 3367 is easily carried out alongside the numerical integration of Step 2. The procedure typically 368 evaluates the noise decomposition in seconds on a desktop computer and may therefore be 369 adequate for statistical inference. 
a. Suppressing intrinsic or extrinsic noise through feedback mechanisms.

Over $40 \%$ of known transcription factors in E. coli regulate their own expression $\underline{40}$. We 372 here investigate the sensitivity of negative autoregulatory feedback to cell cycle fluctuations. 373 We consider transcription and degradation of mRNA molecules from which proteins are 374 synthesised

$$
\begin{aligned}
& \varnothing \stackrel{h(p)}{\longrightarrow} \text { mRNA } \stackrel{k_{1}}{\rightarrow} \varnothing, \\
& \text { mRNA } \stackrel{k_{2}}{\longrightarrow} \text { mRNA + Protein. }
\end{aligned}
$$

The effect of negative feedback is modelled via a Hill-function for the transcription rate 376 $h(p)=\frac{k_{0}}{1+(p / K)^{4}}$, which decreases with the protein number $p$. This approximation is appro- 377 priate when the promoter-binding is extremely fast $\underline{41}$. 378

In Fig. 4 a we show mRNA levels in lineages decrease with cell cycle variability for various 379 feedback strength (the inverse of the dissociation constant, $1 / K$ ). Mean mRNA numbers 380 in the snapshot statistic either decrease (weak, moderate feedback) or increase with cell 381 cycle variability (strong feedback) depending on the feedback strength. In contrast, protein 382 levels increase with cell cycle variability in lineages but decrease in the snapshot for various 383 feedback strengths (Fig. 4b). In agreement with this trend, we find that intrinsic noise 384 always increases with cell cycle variability while the opposite holds for weak to moderate 385 feedback but not for strong feedback (Fig. 4 d). Strikingly, due to the negative feedback 386 regulation, the sensitivity of intrinsic noise of mRNAs is the opposite (Fig. 4c). In contrast 387 to the intrinsic noise properties of the circuit, the total extrinsic noise of the circuit always 388 increases with cell cycle variability, both in lineages and snapshots (Fig. 4 d,e). In all cases, 389 our approximations are in good agreement with exact stochastic simulations (Fig. 4 dots) 390 carried out using the First-Division Algorithm given in Ref ${ }^{31}$ including two non-interacting 391 reporter networks.

Finally, we use the noise decomposition to understand how heterogeneity can be controlled 393 by natural and synthetic circuits. Negative feedback is widely known to reduce noise but 394 often requires fine-tuned parameters 42,44 . How this translates to individual functional noise 395 components, such as intrinsic and extrinsic noise, has only been explored in response to 396 parameter fluctuations $\sqrt[6 \mid 45]{6 u t}$ not in the context of the ubiquitous population dynamics. 397 Here, we are specifically interested in the sensitivity of lineage and population snapshot 398 statistics to cell cycle noise. 
In Fig. 5 a we show that negative feedback can efficiently suppress intrinsic noise as the 400 feedback strength is varied. Intriguingly, comparing the minimum noise levels in lineages and 401 snapshots, vastly different values of the dissociation constants achieve noise suppression in 402 these measures. To study this dependency in more detail, we compute the optimal feedback ${ }_{403}$ strength that minimises intrinsic noise as shown in Fig. 5b. Intriguingly, the optimal values 404 exhibit opposite sensitivities to the cell cycle variability in lineages than in the population 405 snapshots. To efficiently suppress intrinsic noise in a lineage, we must decrease the feedback 406 strength in response to an increase in cell cycle variability. To compensate for intrinsic ${ }_{407}$ variability across the population, however, the feedback strength must increase by almost a 408 two-fold of what would be required in the lineage. 409

In other situations, it may be advantageous to reduce the extrinsic instead of the intrinsic $\quad 410$ noise component. In Fig. 5c, we show that tuning the dissociation constant $(K)$ can similarly ${ }_{411}$ reduce the transmitted cell cycle noise $\mathrm{CV}_{c c}^{2}$. Comparing the optimal feedback strength ${ }_{412}$ $(1 / K)$ as a function of the cell cycle noise $\mathrm{CV}\left[\tau_{d}\right]$ (Fig. $\left.5 \mathrm{~d}\right)$, we observe that they increase with ${ }_{413}$ cell cycle variability in both lineage and snapshot. It is worth noting that vastly different ${ }_{414}$ feedback strengths achieve either intrinsic or extrinsic noise suppression (cf. Fig. 5r,d). ${ }^{415}$ These findings highlight that a single feedback loop may not be sufficient to simultaneously ${ }_{416}$ suppress both noise components whether in lineages or population snapshots. 417

\section{Discussion}

We presented an analytical framework to analyse stochastic biochemical reactions in an ${ }_{419}$ exponentially growing cell population. This theory allows us to characterise and system- ${ }_{420}$ atically decomposes cellular noise into intrinsic and extrinsic components, which applies to ${ }_{421}$ general stochastic biochemical networks. We found that a typical cell in the population ${ }_{422}$ expresses lower levels of proteins per cell than an isolated cell tracked over successive cell ${ }_{423}$ divisions. As a consequence, we observed higher levels of intrinsic noise but, for the exam- ${ }^{424}$ ples studied, the extrinsic noise component was significantly reduced. These effects are most ${ }_{425}$ pronounced in the presence of division time variability as it is the case in natural popula- ${ }_{426}$ tions. Importantly, this highlights that one needs to account for cell cycle fluctuations when ${ }_{427}$ modelling either intrinsic or extrinsic noise components. 428

Previous studies ${ }^{46 \mid 47}$ focussed solely on the effect of age-structure but mostly neglected cell ${ }_{429}$ 
cycle variations. We demonstrated that the statistics of lineages and population snapshots ${ }_{430}$ are not equivalent even when the cell cycle position is known. Although these differences 431 appear to be small when divisions occur deterministically, they will be pronounced in the ${ }_{432}$ presence of division time variability (Figs. 3 and 4). In particular, we showed that measuring 433 cells within a narrow range of cell cycle stages, as for instance achieved through gating 8 does not eliminate all extrinsic noise due to cell cycle fluctuations. In reality, cells are ${ }_{435}$ affected by more than one type of extrinsic noise source as reaction rates may fluctuate over ${ }_{436}$ time and between cells $\$$ [/7/13. These effects should be added to the transmitted extrinsic noise. ${ }_{437}$ We anticipate, however, that it will be difficult in practice to distinguish these fluctuations ${ }_{438}$ from the variations induced by cell cycle variability. 439

While this study focused on the effects of age-structure on biochemical dynamics, several ${ }_{440}$ simulation studies suggest that cell size also coordinates gene expression ${ }^{48 / 49}$. Incorporat- ${ }_{441}$ ing additional physiological details such as cell size into our framework could thus provide ${ }_{442}$ insights to the statistics of intracellular concentrations ${ }^{50}+52$ and extrinsic noise transduced ${ }_{443}$ from cell size control and growth rate fluctuations 53 . A different limitation of this study ${ }_{444}$ is that it is based on the linear noise approximation, which albeit being exact for linear 445 reaction networks, represents an approximation assuming large molecule numbers. Its esti- ${ }_{446}$ mates can be inaccurate for nonlinear reaction networks involving low numbers of molecules. ${ }_{447}$ An improvement to this approximation could employ higher order terms in the system size 448 expansion ${ }^{41 / 54}$, or close the hierarchy of moments using moment closure approximations ${ }^{55}$. $\quad{ }_{449}$

Heterogeneity inferred from snapshots is often used to say something about a cell's history. $\quad 450$ By grouping cells of similar ages, as in ergodic rate analysis $\frac{56}{}$, one can in principle reconstruct 451 time-course information. We demonstrated that such a procedure produces different results ${ }_{452}$ to the lineage statistic (see Fig. 2). Instead, the variability across the population is equivalent ${ }_{453}$ to choosing an arbitrary cell from the final population and tracing it backwards in time ${ }^{31}{ }_{454}$ Although this equivalence provides a sample-path interpretation of snapshot data, it is 455 worth pointing out that it does not apply when cell ages are unidentified. In this case, 456 understanding the relationship between single cell fluctuations and population heterogeneity ${ }_{457}$ requires an agent-based framework as the one presented. 458

We showed that gene expression noise in populations is coupled to the population growth ${ }_{459}$ rate, as observed in population studies 57 . This dependency is crucial when quantifying 460 summary statistics such as mean and variances. We found that the cellular heterogeneity 461 
displays opposite sensitivities to cell cycle variability across populations and lineages. For ${ }_{462}$ negative feedback circuits, this implies that no parameter tuning enables cells to minimise ${ }_{463}^{46}$ noise of both measures efficiently (Fig. 5). Reducing noise in lineages over time comes at 464 the cost of increased population heterogeneity, a strategy cells could exploit to diversify in 465 response to stress ${ }^{58}$. Conversely, tuning snapshot homogeneity sacrifices lineage-optimality, 466 which could confer advantages when gene expression couples to global physiological factors ${ }_{467}$ such as cell size, growth rate or cell division $\underline{59}$.

Cells may thus perform suboptimally depending on which experimental setup is used to 469 study them, whether it is a mother machine or a chemostat. Identifying the relevant noise 470 components and cellular objectives will likely depend on the environmental and experimental 471 conditions, or even on the particular application 58159 . These dependencies thus reveal a 472 fundamental trade-off for the evolution of natural circuits and the design of synthetic circuits 473 in living cells.

In summary, we presented an agent-based framework for the statistical analysis of pop- ${ }^{475}$ ulation snapshots. Inherent in this approach are several noise sources that reveal typical 476 features of snapshot data using noise decompositions. The present framework is widely appli- 477 cable and as such it also applies to large gene regulatory, signalling or metabolic networks. 478 We, therefore, envision that the proposed moment-based approach could prove especially 479 useful for parameter inference from snapshots of living cells 60 .

\section{Supporting information}

\section{A Statistics of interdivision times and age distributions}

We here characterise the statistics of division times and age-distributions in lineages and 483 populations. To this end, it is useful to recall the definition of the Laplace transform of the ${ }_{484}$ interdivision time distribution $\varphi\left(\tau_{d}\right)$ in a lineage

$$
\hat{\varphi}(s)=\int_{0}^{\infty} \mathrm{d} \tau_{d} e^{-s \tau_{d}} \varphi\left(\tau_{d}\right) .
$$

We assume that either $\varphi\left(\tau_{d}\right)$ is known or can be calculated from the division rate via Eq. (7). $\quad 486$ 
To calculate the statistics of the interdivision times in the population, we employ the ${ }_{488}$ Laplace transform of Eq. (11), which is

$$
\hat{\rho}(s)=2 \hat{\varphi}(\lambda+s) .
$$

and note that $\hat{\varphi}(\lambda)=1 / 2$ due to characteristic equation (8). The moments can thus be 490 expressed in terms of the Laplace transform

$$
E_{\rho}\left[\tau_{d}^{n}\right]=2\left(-\frac{\partial}{\partial \lambda}\right)^{n} \hat{\varphi}(\lambda)
$$

From these, we can compute

$$
E_{\rho}\left[\tau_{d}\right]=-2 \hat{\varphi}^{\prime}(\lambda), \quad \operatorname{Var}_{\rho}\left[\tau_{d}\right]=2 \hat{\varphi}^{\prime \prime}(\lambda)-4 \hat{\varphi}^{\prime}(\lambda)^{2}
$$

and hence

$$
\mathrm{CV}_{\rho}^{2}\left[\tau_{d}\right]=\frac{\hat{\varphi}^{\prime \prime}(\lambda)}{2 \hat{\varphi}^{\prime}(\lambda)^{2}}-1
$$

\section{Age-distribution in lineages}

The age-distribution yields the frequency of cell ages observed for different single cell measures. To compute the moments of the age-distribution in a lineage, we compute the Laplace transform of Eq. (13), which gives

$$
\hat{\pi}(s)=\frac{1-\hat{\varphi}(s)}{s E_{\varphi}\left[\tau_{d}\right]} .
$$

By differentiating the above expression repeatedly at $s=0$, we find

$$
E_{\pi}[\tau]=\frac{E_{\varphi}\left[\tau^{2}\right]}{2 E_{\varphi}[\tau]}, \quad \operatorname{Var}_{\pi}[\tau]=\frac{E_{\varphi}\left[\tau^{3}\right]}{3 E_{\varphi}[\tau]}
$$

and

$$
\mathrm{CV}_{\pi}^{2}[\tau]=\frac{4 E_{\varphi}[\tau] E_{\varphi}\left[\tau^{3}\right]}{3 E_{\varphi}\left[\tau^{2}\right]}
$$

which concludes the the age-statistics in the population snapshot. 


\section{Age-distribution in populations}

Similarly, we consider the Laplace transform of the age-distribution in a population snap- 502 shot, Eq. (6), which evaluates to

$$
\begin{aligned}
\hat{\Pi}(s)=E_{\Pi}\left[e^{-s \tau}\right] & =2 \lambda \int_{0}^{\infty} \mathrm{d} \tau \Phi(\tau) e^{-(s+\lambda) \tau} \\
& =\frac{2 \lambda}{s+\lambda}(1-\hat{\varphi}(s+\lambda)),
\end{aligned}
$$

Repeated differentiation at $s=0$, gives

$$
E_{\Pi}[\tau]=\frac{1}{\lambda}+2 \hat{\varphi}^{\prime}(\lambda), \quad \operatorname{Var}_{\Pi}[\tau]=\frac{1}{\lambda^{2}}-2 \hat{\varphi}^{\prime \prime}(\lambda)-4 \hat{\varphi}^{\prime}(\lambda)^{2}
$$

It is straightforward to evaluate these statistics numerically as we do for the log-normal 505 distribution in Fig. 2 and 3. For the gamma-distribution, the population growth rate, the 506 age- and interdivision-time distributions can be obtained in close form as we show in the 507 following.

4 Gamma distribution: Explicit solutions to population growth rate and the age/interdivision 509 time distributions

We fix the division time to be gamma distributed with density function

$$
\varphi\left(\tau_{d}\right)=\frac{e^{-\frac{\tau_{d}}{c \mu}}\left(\frac{\tau_{d}}{c \mu}\right) 1 / c}{\Gamma\left(\frac{1}{c}\right) \tau_{d}},
$$

where $\Gamma$ is the gamma function, such that $E_{\varphi}\left[\tau_{d}\right]=\mu$ and $c=\operatorname{CV}_{\varphi}^{2}\left[\tau_{d}\right]$. The Laplace ${ }_{512}$ transform of the distribution is

$$
\hat{\varphi}(s)=(c \mu s+1)^{-1 / c} .
$$

Recasting now the Euler-Lotka equation in the form $\hat{\varphi}(\lambda)=1 / 2$, we can solve for $\lambda$ to obtain 514

$$
\lambda=\frac{2^{c}-1}{c \mu} .
$$

The division time distribution in the population is then given by

$$
\rho\left(\tau_{d}\right)=2 e^{-\lambda \tau_{d}} \varphi\left(\tau_{d}\right)=\frac{2 e^{-\frac{2^{c} \tau_{d}}{c \mu}}\left(\frac{\tau_{d}}{c \mu}\right) 1 / c}{\Gamma\left(\frac{1}{c}\right) \tau_{d}},
$$


which is also a gamma distribution but with a shorter mean division time but the same 516 coefficient of variation

$$
E_{\rho}\left[\tau_{d}\right]=2^{-c} \mu, \quad \mathrm{CV}_{\rho}^{2}\left[\tau_{d}\right]=c .
$$

Further, the age-distribution in a lineage is

$$
\pi(\tau)=\frac{1}{\mu} \frac{\Gamma\left(\frac{1}{c}, \frac{\tau}{c \mu}\right)}{\Gamma\left(\frac{1}{c}\right)},
$$

where $\Gamma(\cdot, \cdot)$ is the upper incomplete gamma function. Its statistics are with statistics

$$
E_{\pi}[\tau]=\frac{\mu}{2}(1+c), \quad \mathrm{CV}_{\pi}^{2}[\tau]=\frac{5 c+1}{3 c+3} .
$$

Similarly, the age-distribution in the population becomes

$$
\Pi(\tau)=2 \lambda e^{-\lambda \tau} \frac{\Gamma\left(\frac{1}{c}, \frac{\tau}{c \mu}\right)}{\Gamma\left(\frac{1}{c}\right)}
$$

with statistics

$$
\begin{aligned}
& E_{\Pi}[\tau]=\mu\left(\frac{c}{2^{c}-1}-\frac{1}{2^{c}}\right) \\
& \mathrm{CV}_{\Pi}^{2}[\tau]=\frac{2 c}{c+2^{-c}-1}-\frac{\left(2^{c}-1\right)^{2}(c+1)}{\left(2^{c}(c-1)+1\right)^{2}}-1 .
\end{aligned}
$$

Interestingly, it follows that $E_{\Pi}[\tau]<E_{\pi}[\tau]$, but $\mathrm{CV}_{\Pi}^{2}[\tau]>\mathrm{CV}_{\pi}^{2}[\tau]$ for $c<1$ and $\mathrm{CV}_{\Pi}^{2}[\tau]<{ }_{522}$ $\mathrm{CV}_{\pi}^{2}[\tau]$ for $c>1$.

\section{B An explict formula for the uncertainty due to unknown cell age}

We here verify Eq. (31c) of the main text, which holds for linear reaction networks. To 525 this end we define $\epsilon(\tau)=E_{\Pi}[x \mid \tau]-E_{\Pi}[x]$ such that $\bar{\Sigma}_{\text {age }}=E_{\Pi}\left[\epsilon \epsilon^{T}\right]$ and use Eq. (18a) to ${ }_{526}$ write

$$
\frac{\partial}{\partial \tau} \epsilon(\tau)=\nu w(E[x \mid \tau])=c+\mathcal{J}\left(\epsilon(\tau)+E_{\Pi}[x]\right)
$$

We used the fact that $\nu w(E[x \mid \tau])=c+\mathcal{J} E[x \mid \tau]$, where $c$ is a constant vector, since for ${ }_{528}$ linear reaction networks the propensities are linear in the number of molecules. Making use ${ }_{529}$ of Eq. (53) we then compute

$$
\begin{aligned}
E_{\Pi}\left[\frac{\partial}{\partial \tau} \epsilon(\tau) \epsilon^{T}(\tau)\right] & =E_{\Pi}\left[\epsilon(\tau) \frac{\partial}{\partial \tau} \epsilon^{T}(\tau)\right]+E_{\Pi}\left[\epsilon^{T}(\tau) \frac{\partial}{\partial \tau} \epsilon(\tau)\right] \\
& =\mathcal{J} \bar{\Sigma}_{\text {age }}+\bar{\Sigma}_{\text {age }} \mathcal{J}^{T} .
\end{aligned}
$$


On the other hand, using Eq. (15) of the main text, it follows that

$$
\begin{aligned}
E_{\Pi}\left[\frac{\partial}{\partial \tau} \epsilon(\tau) \epsilon^{T}(\tau)\right] & =\lambda\left(\bar{\Sigma}_{\text {age }}+E_{\rho}\left[\epsilon(\tau) \epsilon^{T}(\tau)\right]-2 \epsilon(0) \epsilon^{T}(0)\right) \\
& =\lambda\left(\bar{\Sigma}_{\text {age }}+\operatorname{Cov}_{\rho}[E[x \mid \tau]]-E_{\Pi}[x] E_{\Pi}\left[x^{T}\right]+2 E_{\rho}[x \mid 0] E_{\rho}\left[x^{T} \mid 0\right]\right) .
\end{aligned}
$$

Combining the last two equation gives the result $31 \mathrm{c}$ ) of the main text.

\section{Detailed discussion of the moment-closure conditions}

While the moment equations derived in Sec. II B are exact, the equations for cells of the ${ }_{534}$ same age are only closed when $w_{r}(x)$ depends at most linearly on the molecule numbers $x \quad 535$ and the covariance of the partitioning kernel $\operatorname{Cov}_{B}\left[x \mid x^{\prime}\right]$ depends at most quadratically on the 536 number molecules in the mother cell $x^{\prime}$. This holds, for instance, for biochemical composed ${ }_{537}$ solely from unimolecular reactions and independent binomial partitioning. Similarly, it 538 holds true for the mean of cells with unknown age, but not generally for their corresponding 539 variances. Specifically, the covariance for cells of unknown age also depends on the moments 540 for cells of known age and thus they must explicitly depend on the division time distribution. $\quad 541$

There are now two scenarios in which the variances are independent of the division time 542 distribution. The first case is when the age-distribution coincides with the division time 543 distribution $\Pi(\tau)=\rho(\tau)$, which follows only when the division rate $\gamma$ is constant and 544 independent of age, i.e. the division times are exponentially distributed. The second case 545 assumes a particular division kernel $B\left(x \mid x^{\prime}\right)$ that satisfies $\operatorname{Cov}_{B}\left[x \mid x^{\prime}\right]=\frac{1}{4} x^{\prime} x^{\prime T}$, which follows ${ }_{546}$ when all molecules are inherited by only one of the daughter cells. In all other cases, which ${ }_{547}$ seem most relevant in practice, the moment equations for unknown cell age involve the 548 moments for cells of known age. Thus, for general nonlinear reaction networks, they involve 549 two hierarchies of moments that cannot be easily closed. A simple and generally applicable 550 approximation that circumvents this problem is given in Sec. III A using the linear noise 551 approximation. 


\section{Analytical noise decomposition for gene expression with degradation}

We consider a simple system in which a protein $P$ is translated in stochastic bursts of ${ }_{554}$ size $m$ and is subsequently degraded

$$
\varnothing \stackrel{k_{0}}{\rightarrow} m \times P, \quad P \stackrel{k_{1}}{\rightarrow} \varnothing .
$$

Because the burst size is a random variable we can recast the synthesis reaction into a series 556 of reactions with reaction rates $k_{0} \pi(m)$,

$$
\begin{aligned}
& \varnothing \stackrel{k_{0} \pi(1)}{\longrightarrow} 1 \times P, \quad \varnothing \stackrel{k_{0} \pi(2)}{\longrightarrow} 2 \times P, \quad \varnothing \stackrel{k_{0} \pi(3)}{\longrightarrow} 3 \times P, \quad \ldots, \\
& P \stackrel{k_{1}}{\longrightarrow} \varnothing
\end{aligned}
$$

where $\pi(m)$ is the distribution of burst sizes, which is geometric for the two-stage model of 558 gene expression.

The equation for the mean number of molecules is then

$$
\frac{\partial E_{\Pi}[x \mid \tau]}{\partial \tau}=k_{0}\left(\sum_{m=1} m \pi(m)\right)-k_{1} E_{\Pi}[x \mid \tau]=b k_{0}-k_{1} E_{\Pi}[x \mid \tau]
$$

with solution

$$
E_{\Pi}[x \mid \tau]=E_{\Pi}[x \mid 0] e^{-k_{1} \tau}+\frac{b k_{0}}{k_{1}}\left(1-e^{-k_{1} \tau}\right)
$$

Substituting the solution into the boundary condition $E_{\rho}\left(E_{\Pi}[x \mid \tau]\right)=2 E_{\Pi}[x \mid 0]$ and solving 563 for $E_{\Pi}[x \mid 0]$ yields the final result

$$
E_{\Pi}[x \mid \tau]=\frac{b k_{0}}{k_{1}}\left(1-\frac{e^{-k_{1} \tau}}{2-\hat{\rho}\left(k_{1}\right)}\right),
$$

where $\hat{\rho}$ is the Laplace transform of the division time distribution

$$
\hat{\rho}(s)=\int_{0}^{\infty} \mathrm{d} \tau_{d} e^{-s \tau_{d}} \rho\left(\tau_{d}\right)
$$

As we will show in the following the statistics of gene expression in growing populations 566 depends crucially on this function. 
To compute the protein fluctuations, we see from (58), that the Jacobian is

$$
\mathcal{J}=-k_{1}
$$

The diffusion matrix then follows from Eq. (19) then follows

$$
\mathcal{D}=k_{0} \sum_{m=1}^{\infty} m^{2} \pi(m)+k_{1} E_{\Pi}[x \mid \tau]=k_{0} b(2 b+1)+k_{1} E_{\Pi}[x \mid \tau] .
$$

The variance of intrinsic and extrinsic fluctuations obeys Eqs. 18b, which read explicitly 571

$$
\begin{gathered}
\frac{\partial}{\partial \tau} \Sigma_{\text {int }}(\tau)=-2 k_{1} \Sigma_{\text {int }}(\tau)+k_{0} b(2 b+1)+k_{1} E_{\Pi}[x \mid \tau] \\
\frac{\partial}{\partial \tau} \Sigma_{\text {ext }}(\tau)=-2 k_{1} \Sigma_{\text {ext }}(\tau) \\
\Sigma_{\text {int }}(\tau)=\frac{b k_{0} e^{-k_{1} t}}{k_{1}\left(g\left(k_{1}\right)-2\right)}+\frac{b(b+1) k_{0}}{k_{1}} \\
+e^{-2 k_{1} t}\left(\frac{b k_{0}\left(-(b+1) g\left(k_{1}\right)+2 b+1\right)}{k_{1}\left(g\left(k_{1}\right)-2\right)}+\Sigma_{\text {int }}(0)\right) \\
\Sigma_{\text {ext }}(\tau)=\Sigma_{\text {ext }}(0) e^{-2 k_{1} t},
\end{gathered}
$$

where $\Sigma_{\text {int }}(0)$ and $\Sigma_{\text {ext }}(0)$ are the intrinsic and extrinsic variation at cell division, which 572 have to be determined from the boundary conditions. According to Eq. (28c) and (28d), 573 the boundary conditions are

$$
\begin{aligned}
& 4 \Sigma_{\text {int }}(0)=E_{\rho}\left[\Sigma_{\text {int }}\left(\tau_{d}\right)\right]+E_{\rho}\left[E_{\Pi}\left[x \mid \tau_{d}\right]\right] \\
& 4 \Sigma_{\text {ext }}(0)=E_{\rho}\left[\Sigma_{\text {ext }}\left(\tau_{d}\right)\right]+\operatorname{Cov}_{\rho}\left[E_{\Pi}\left[x \mid \tau_{d}\right], E_{\Pi}\left[x \mid \tau_{d}\right]\right] .
\end{aligned}
$$

To compute these values we notice that the variances at cell division follow from averaging 575 Eqs. (64) over the division time distribution $\rho$, which results in

$$
\begin{aligned}
E_{\rho}\left[\Sigma_{\text {int }}\left(\tau_{d}\right)\right] & =\frac{b k_{0} \hat{\rho}\left(k_{1}\right)}{k_{1}\left(g\left(k_{1}\right)-2\right)}+\frac{b(b+1) k_{0}}{k_{1}} \\
+\hat{\rho}\left(2 k_{1}\right) & \left(\frac{b k_{0}\left(-(b+1) g\left(k_{1}\right)+2 b+1\right)}{k_{1}\left(g\left(k_{1}\right)-2\right)}+\Sigma_{\text {int }}(0)\right) \\
E_{\rho}\left[\Sigma_{\text {ext }}\left(\tau_{d}\right)\right] & =\Sigma_{\text {ext }}(0) \hat{\rho}\left(2 k_{1}\right) .
\end{aligned}
$$


Further, we evaluate

$$
E_{\rho}\left(E_{\Pi}\left[x \mid \tau_{d}\right]\right)=2 E_{\Pi}[x \mid 0]=2 \frac{b k_{0}}{k_{1}}\left(\frac{1-\hat{\rho}\left(k_{1}\right)}{2-\hat{\rho}\left(k_{1}\right)}\right)
$$

and

$$
\begin{aligned}
\operatorname{Cov}_{\rho}\left[E_{\Pi}\left[x \mid \tau_{d}\right], E_{\Pi}\left[x \mid \tau_{d}\right]\right] & =E_{\rho}\left(E_{\Pi}[x \mid \tau]-E_{\rho}\left(E_{\Pi}[x \mid \tau]\right)\right)^{2} \\
& =\left(\frac{b k_{0}}{k_{1}\left(2-\hat{\rho}\left(k_{1}\right)\right)}\right)^{2} E_{\rho}\left(\hat{\rho}\left(k_{1}\right)-e^{-k_{1} \tau}\right)^{2} \\
& =\left(\frac{b k_{0}}{k_{1}\left(2-\hat{\rho}\left(k_{1}\right)\right)}\right)^{2}\left(\hat{\rho}\left(2 k_{1}\right)-\hat{\rho}^{2}\left(k_{1}\right)\right) .
\end{aligned}
$$

Plugging Eqs. 67), 68) and (69) into (66), solving for $\Sigma_{\text {int }}(0)$ and $\Sigma_{\text {ext }}(0)$ and using the 579 result in Eqs. 64, we finally arrive at

$$
\begin{aligned}
& \Sigma_{\text {int }}(\tau)=\frac{3 b^{2} k_{0} e^{-2 k_{1} t}}{k_{1}\left(\hat{\rho}\left(2 k_{1}\right)-4\right)}+\frac{b k_{0} e^{-k_{1} t}}{k_{1}\left(\hat{\rho}\left(k_{1}\right)-2\right)}+\frac{(b+1) b k_{0}}{k_{1}}, \\
& \Sigma_{\text {ext }}(\tau)=\frac{b^{2} k_{0}^{2}\left(\hat{\rho}\left(2 k_{1}\right)-\hat{\rho}^{2}\left(k_{1}\right)\right) e^{-2 k_{1} t}}{k_{1}^{2}\left(\hat{\rho}\left(k_{1}\right)-2\right)^{2}\left(4-\hat{\rho}\left(2 k_{1}\right)\right)},
\end{aligned}
$$

which determines the progression of intrinsic and extrinsic fluctuations over the cell cycle. $\quad{ }^{581}$

\section{Protein statistics for cells of unknown age}

The mean protein number is given by

$$
E_{\Pi}[x]=\frac{b k_{0}}{k_{1}+\lambda} .
$$

Thus the mean number is determined from the balance between the rates of translation, 584 degradation and dilution due to cell divisions. From Eqs. (31) we compute

$$
\begin{aligned}
& \bar{\Sigma}_{\text {int }}=\frac{b(b+1) k_{0}}{k_{1}}-\frac{b \lambda k_{0}}{\lambda k_{1}+k_{1}^{2}}+\frac{3 b^{2} \lambda k_{0}\left(\hat{\rho}\left(2 k_{1}\right)-2\right)}{k_{1}\left(4-\hat{\rho}\left(2 k_{1}\right)\right)\left(\lambda+2 k_{1}\right)}, \\
& \bar{\Sigma}_{\text {ext }}=\frac{b^{2} \lambda k_{0}^{2}\left(\hat{\rho}\left(2 k_{1}\right)-\hat{\rho}^{2}\left(k_{1}\right)\right)\left(2-\hat{\rho}\left(2 k_{1}\right)\right)}{k_{1}^{2}\left(2-\hat{\rho}\left(k_{1}\right)\right)^{2}\left(4-\hat{\rho}\left(2 k_{1}\right)\right)\left(\lambda+2 k_{1}\right)} \\
& \bar{\Sigma}_{\text {age }}=\frac{b^{2} \lambda k_{0}^{2}\left(2-\hat{\rho}\left(2 k_{1}\right)\right)}{k_{1}^{2}\left(\hat{\rho}\left(k_{1}\right)-2\right)^{2}\left(\lambda+2 k_{1}\right)}-\frac{b^{2} \lambda^{2} k_{0}^{2}}{k_{1}^{2}\left(\lambda+k_{1}\right)^{2}} .
\end{aligned}
$$

Finally, we compute $\mathrm{CV}_{y}^{2}=\bar{\Sigma}_{y} / E_{\Pi}[x]^{2}$ to arrive at the expressions for the coefficient of ${ }_{586}$ 
variations

$$
\begin{aligned}
\mathrm{CV}_{\text {int }}^{2} & =\frac{1}{E_{\Pi}[x]}\left(1+b\left(1+\frac{b \lambda}{k_{1}}\right)-\frac{3 b \lambda}{k_{1}} \frac{\left(2-\hat{\rho}\left(2 k_{1}\right)\right)}{\left(4-\hat{\rho}\left(2 k_{1}\right)\right)} \frac{\left(\lambda+k_{1}\right)}{\left(\lambda+2 k_{1}\right)}\right), \\
\mathrm{CV}_{\mathrm{cc}}^{2} & =\frac{\lambda\left(\hat{\rho}\left(2 k_{1}\right)-\hat{\rho}\left(k_{1}\right)^{2}\right)\left(\hat{\rho}\left(2 k_{1}\right)-2\right)\left(\lambda+k_{1}\right)^{2}}{k_{1}^{2}\left(\hat{\rho}\left(k_{1}\right)-2\right)^{2}\left(\hat{\rho}\left(2 k_{1}\right)-4\right)\left(\lambda+2 k_{1}\right)}, \\
\mathrm{CV}_{\text {age }}^{2} & =\frac{\lambda\left(2-\hat{\rho}\left(2 k_{1}\right)\right)\left(\lambda+k_{1}\right)^{2}}{k_{1}^{2}\left(\hat{\rho}\left(k_{1}\right)-2\right)^{2}\left(\lambda+2 k_{1}\right)}-\frac{\lambda^{2}}{k_{1}^{2}},
\end{aligned}
$$

which denote the intrinsic noise, the transmitted noise from cell cycle fluctuations and the 588 uncertainty due to the unknown cell age. It is obvious that these expressions are much 589 more involved than for the case without degradation because they depend on the Laplace 590 transform $\hat{\rho}$ of the division time distribution. Interestingly, the last two components, which 591 together represent the extrinsic noise, also depend on the degradation rate $k_{1}$ meaning that 592 the total extrinsic noise is constant only when measured the mean concentration is varied 593 through the either transcription rate or burst size.

\section{Acknowledgments}

It is a pleasure to thank Vahid Shahrezaei for valuable feedback.

\section{Funding}

PT acknowledges a fellowship by The Royal Commission for the Exhibition of 1851 and 598 support by the EPSRC Centre for Mathematics of Precision Healthcare (EP/N014529/1). 599

\section{References}

${ }^{1}$ M. B. Elowitz, A. J. Levine, E. D. Siggia, and P. S. Swain, "Stochastic gene expression in a single cell," Science 297, 1183-1186 (2002).

${ }^{2}$ J. M. Raser and E. K. O'shea, "Noise in gene expression: origins, consequences, and control," Science 309, 2010-2013 (2005).

${ }^{3}$ J. M. Raser and E. K. O'shea, "Control of stochasticity in eukaryotic gene expression," Science 304, 1811-1814 (2004). 
${ }^{4}$ C. V. Harper, B. Finkenstädt, D. J. Woodcock, S. Friedrichsen, S. Semprini, L. Ashahrezaei2008ll, D. G. Spiller, J. J. Mullins, D. A. Rand, J. R. Davis, et al., "Dynamic analysis of stochastic transcription cycles," PLoS Biol 9, e1000607 (2011).

${ }^{5}$ I. S. Araújo, J. M. Pietsch, E. M. Keizer, B. Greese, R. Balkunde, C. Fleck, and M. Hülskamp, "Stochastic gene expression in Arabidopsis thaliana," Nat Commun 8, 2132 $(2017)$.

${ }^{6}$ V. Shahrezaei, J. F. Ollivier, and P. S. Swain, "Colored extrinsic fluctuations and stochastic gene expression," Mol Syst Biol 4, 196 (2008).

${ }^{7}$ A. Hilfinger and J. Paulsson, "Separating intrinsic from extrinsic fluctuations in dynamic biological systems," Proc Natl Acad Sci 108, 12167-12172 (2011).

${ }^{8}$ C. Zechner, J. Ruess, P. Krenn, S. Pelet, M. Peter, J. Lygeros, and H. Koeppl, "Momentbased inference predicts bimodality in transient gene expression," Proc Natl Acad Sci 109, 8340-8345 (2012).

${ }^{9}$ C. G. Bowsher, M. Voliotis, and P. S. Swain, "The fidelity of dynamic signaling by noisy biomolecular networks," PLoS Comput Biol 9, e1002965 (2013).

${ }^{10}$ A. Singh and M. Soltani, "Quantifying intrinsic and extrinsic variability in stochastic gene expression models," PLoS One 8, e84301 (2013).

${ }^{11}$ J. Hasenauer, C. Hasenauer, T. Hucho, and F. J. Theis, "Ode constrained mixture modelling: a method for unraveling subpopulation structures and dynamics," PLoS Comput Biol 10, e1003686 (2014).

${ }^{12}$ M. Voliotis, P. Thomas, R. Grima, and C. G. Bowsher, "Stochastic simulation of biomolecular networks in dynamic environments," PLoS Comput Biol 12, e1004923 (2016).

${ }^{13} \mathrm{~J}$. Dattani and M. Barahona, "Stochastic models of gene transcription with upstream drives: exact solution and sample path characterization," J Royal Soc Interface 14, 20160833 (2017).

${ }^{14}$ M. Del Giudice, S. Bo, S. Grigolon, and C. Bosia, "On the role of extrinsic noise in microrna-mediated bimodal gene expression," PLoS Comput Biol 14, e1006063 (2018).

${ }^{15}$ D. Volfson, J. Marciniak, W. J. Blake, N. Ostroff, L. S. Tsimring, and J. Hasty, "Origins of extrinsic variability in eukaryotic gene expression," Nature 439, 861-864 (2006).

${ }^{16}$ M. Hashimoto, T. Nozoe, H. Nakaoka, R. Okura, S. Akiyoshi, K. Kaneko, E. Kussell, and Y. Wakamoto, "Noise-driven growth rate gain in clonal cellular populations," Proc Natl Acad Sci , 201519412 (2016). 
${ }^{17}$ B. Cerulus, A. M. New, K. Pougach, and K. J. Verstrepen, "Noise and epigenetic inheritance of single-cell division times influence population fitness," Curr Biol 26, 1138-1147 (2016).

${ }^{18}$ F. Shen, L. Hodgson, A. Rabinovich, O. Pertz, K. Hahn, and J. H. Price, "Functional proteometrics for cell migration," Cytometry Part A 69, 563-572 (2006).

${ }^{19}$ P. S. Swain, M. B. Elowitz, and E. D. Siggia, "Intrinsic and extrinsic contributions to stochasticity in gene expression," Proc Natl Acad Sci 99, 12795-12800 (2002).

${ }^{20}$ J. Lloyd-Price, H. Tran, and A. S. Ribeiro, "Dynamics of small genetic circuits subject to stochastic partitioning in cell division," J Theor Biol 356, 11-19 (2014).

${ }^{21}$ V. Bierbaum and S. Klumpp, "Impact of the cell division cycle on gene circuits," Phys Biol 12, 066003 (2015).

${ }^{22}$ I. G. Johnston and N. S. Jones, "Closed-form stochastic solutions for non-equilibrium dynamics and inheritance of cellular components over many cell divisions," Proc Royal Soc A 471, 20150050 (2015).

${ }^{23}$ M. Soltani, C. A. Vargas-Garcia, D. Antunes, and A. Singh, "Intercellular variability in protein levels from stochastic expression and noisy cell cycle processes," PLoS Comput Biol 12, e1004972 (2016).

${ }^{24}$ A. Roy and S. Klumpp, "Simulating genetic circuits in bacterial populations with growth heterogeneity," Biophys J 114, 484-492 (2018).

${ }^{25}$ P. Wang, L. Robert, J. Pelletier, W. L. Dang, F. Taddei, A. Wright, and S. Jun, "Robust growth of Escherichia coli," Curr Biol 20, 1099-1103 (2010).

${ }^{26}$ J. C. Locke and M. B. Elowitz, "Using movies to analyse gene circuit dynamics in single cells," Nat Rev Microbiol 7, 383 (2009).

${ }^{27}$ D. Muzzey and A. van Oudenaarden, "Quantitative time-lapse fluorescence microscopy in single cells," Annu Rev Cell Dev Biol 25, 301-327 (2009).

${ }^{28}$ J. R. Newman, S. Ghaemmaghami, J. Ihmels, D. K. Breslow, M. Noble, J. L. DeRisi, and J. S. Weissman, "Single-cell proteomic analysis of S. cerevisiae reveals the architecture of biological noise," Nature 441, 840 (2006).

${ }^{29}$ L.-h. So, A. Ghosh, C. Zong, L. A. Sepúlveda, R. Segev, and I. Golding, "General properties of transcriptional time series in Escherichia coli," Nat Genet 43, 554 (2011).

${ }^{30}$ B. Okumus, D. Landgraf, G. C. Lai, S. Bakshi, J. C. Arias-Castro, S. Yildiz, D. Huh, R. Fernandez-Lopez, C. N. Peterson, E. Toprak, et al., "Mechanical slowing-down of cy- 
toplasmic diffusion allows in vivo counting of proteins in individual cells," Nat Commun 7, 11641 (2016).

${ }^{31} \mathrm{P}$. Thomas, "Making sense of snapshot data: ergodic principle for clonal cell populations," J Royal Soc Interface 14, 20170467 (2017).

${ }^{32}$ E. Powell, "Growth rate and generation time of bacteria, with special reference to continuous culture," Microbiology 15, 492-511 (1956).

${ }^{33}$ P. Painter and A. Marr, "Mathematics of microbial populations," Annu Rev Microbiol 22, 519-548 (1968).

${ }^{34}$ Y. Wakamoto, A. Y. Grosberg, and E. Kussell, "Optimal lineage principle for agestructured populations," Evolution 66, 115-134 (2012).

${ }^{35}$ N. G. Van Kampen, Stochastic processes in physics and chemistry (Elsevier, 1992).

${ }^{36}$ J. Elf and M. Ehrenberg, "Fast evaluation of fluctuations in biochemical networks with the linear noise approximation," Genome Res 13, 2475-2484 (2003).

${ }^{37}$ V. Shahrezaei and P. S. Swain, "Analytical distributions for stochastic gene expression," Proc Natl Acad Sci 105, 17256-17261 (2008).

${ }^{38}$ C. G. Bowsher and P. S. Swain, "Identifying sources of variation and the flow of information in biochemical networks," Proc Natl Acad Sci 109, E1320-E1328 (2012).

${ }^{39}$ D. Huh and J. Paulsson, "Random partitioning of molecules at cell division," Proc Natl Acad Sci 108, 15004-15009 (2011).

${ }^{40}$ D. Thieffry, A. M. Huerta, E. Pérez-Rueda, and J. Collado-Vides, "From specific gene regulation to genomic networks: a global analysis of transcriptional regulation in Escherichia coli," Bioessays 20, 433-440 (1998).

${ }^{41} \mathrm{P}$. Thomas and R. Grima, "Approximate probability distributions of the master equation," Phys Rev E 92, 012120 (2015).

${ }^{42}$ N. Rosenfeld, M. B. Elowitz, and U. Alon, "Negative autoregulation speeds the response times of transcription networks," J Mol Biol 323, 785-793 (2002).

${ }^{43}$ Y. Dublanche, K. Michalodimitrakis, N. Kümmerer, M. Foglierini, and L. Serrano, "Noise in transcription negative feedback loops: simulation and experimental analysis," Mol Syst Biol 2, 41 (2006).

${ }^{44}$ D. A. Oyarzun, J.-B. Lugagne, and G.-B. V. Stan, "Noise propagation in synthetic gene circuits for metabolic control," ACS Synth Biol 4, 116-125 (2014). 
${ }^{45}$ A. Singh and J. P. Hespanha, "Optimal feedback strength for noise suppression in autoregulatory gene networks," Biophys J 96, 4013-4023 (2009).

${ }^{46}$ O. G. Berg, "A model for the statistical fluctuations of protein numbers in a microbial population," J Theor Biol 71, 587-603 (1978).

${ }^{47}$ A. Schwabe and F. J. Bruggeman, "Contributions of cell growth and biochemical reactions to nongenetic variability of cells," Biophys J 107, 301-313 (2014).

${ }^{48}$ F. Bertaux, S. Marguerat, and V. Shahrezaei, "Division rate, cell size and proteome allocation: impact on gene expression noise and implications for the dynamics of genetic circuits," Royal Soc Open Sci 5, 172234 (2018).

${ }^{49}$ C. Vargas-Garcia, K. Ghusinga, and A. Singh, "Cell size control and gene expression homeostasis in single-cells." Curr Opin Syst Biol 8, 109-116 (2018).

${ }^{50}$ T. Blasi, F. Buettner, M. K. Strasser, C. Marr, and F. J. Theis, "cgcorrect: a method to correct for confounding cell-cell variation due to cell growth in single-cell transcriptomics," Phys Biol 14, 036001 (2017).

${ }^{51}$ J. H. Heerden, H. Kempe, A. Doerr, T. Maarleveld, N. Nordholt, and F. J. Bruggeman, "Statistics and simulation of growth of single bacterial cells: illustrations with B. subtilis and E. coli," Sci Rep 7, 16094 (2017).

${ }^{52} \mathrm{P}$. Thomas, "Analysis of cell size homeostasis at the single-cell and population level," Frontiers in Physics 6, 64 (2018).

${ }^{53}$ S. Tsuru, J. Ichinose, A. Kashiwagi, B.-W. Ying, K. Kaneko, and T. Yomo, "Noisy cell growth rate leads to fluctuating protein concentration in bacteria," Phys Biol 6, 036015 (2009).

${ }^{54}$ R. Grima, P. Thomas, and A. V. Straube, "How accurate are the nonlinear chemical Fokker-Planck and chemical Langevin equations?" J Chem Phys 135, 084103 (2011).

${ }^{55}$ D. Schnoerr, G. Sanguinetti, and R. Grima, "Comparison of different moment-closure approximations for stochastic chemical kinetics," J Chem Phys 143, 11B610_1 (2015).

${ }^{56}$ R. Kafri, J. Levy, M. B. Ginzberg, S. Oh, G. Lahav, and M. W. Kirschner, "Dynamics extracted from fixed cells reveal feedback linking cell growth to cell cycle," Nature 494, 480-483 (2013).

${ }^{57}$ L. Keren, D. Van Dijk, S. Weingarten-Gabbay, D. Davidi, G. Jona, A. Weinberger, R. Milo, and E. Segal, "Noise in gene expression is coupled to growth rate," Genome Res 25, 18931902 (2015). 
${ }^{58}$ C. van Boxtel, J. H. van Heerden, N. Nordholt, P. Schmidt, and F. J. Bruggeman, "Taking chances and making mistakes: non-genetic phenotypic heterogeneity and its consequences for surviving in dynamic environments," J Royal Soc Interface 14, 20170141 (2017).

${ }^{59}$ B. M. Martins and J. C. Locke, "Microbial individuality: how single-cell heterogeneity enables population level strategies," Curr Opin Microbiol 24, 104-112 (2015).

${ }^{60}$ F. Fröhlich, P. Thomas, A. Kazeroonian, F. J. Theis, R. Grima, and J. Hasenauer, "Inference for stochastic chemical kinetics using moment equations and system size expansion," PLoS Comput Biol 12, e1005030 (2016). 
(a) agent-based population

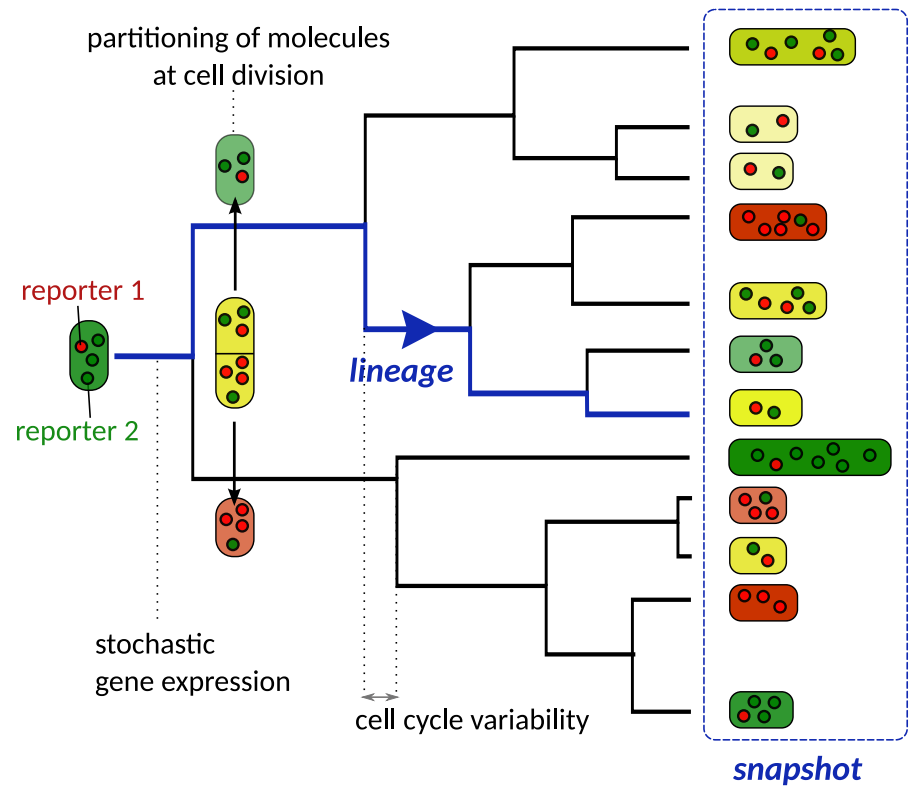

(b) population

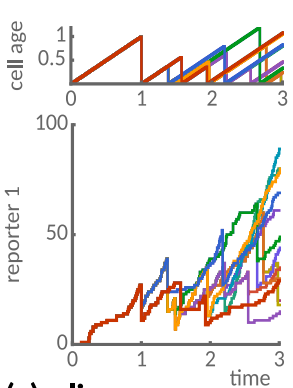

(c) lineage
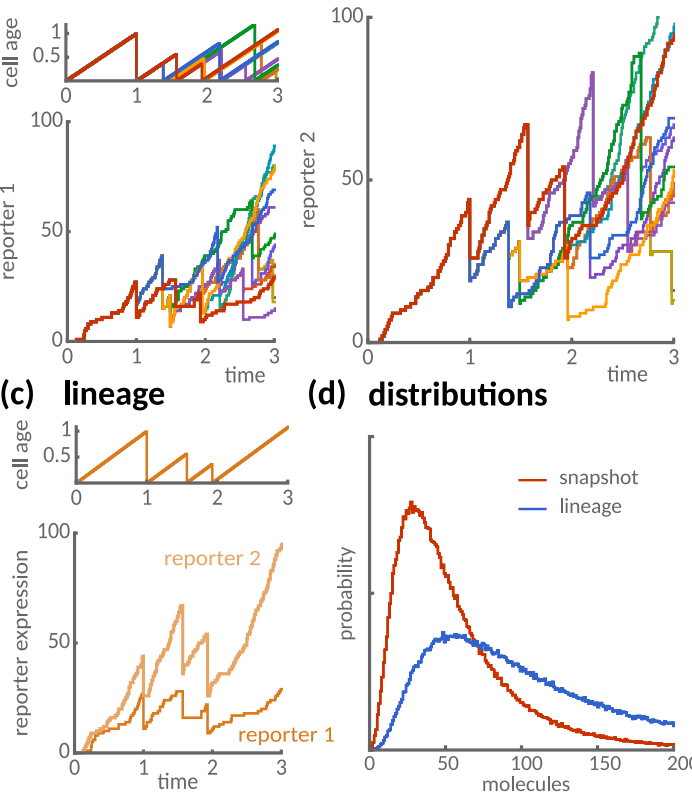

(d) distributions

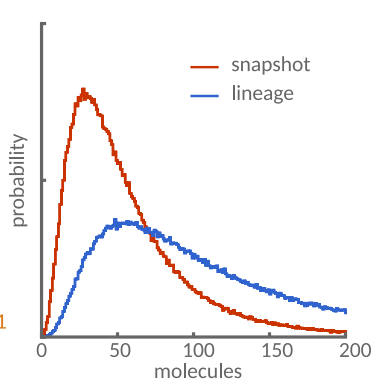

FIG. 1. Agent-based model of clonal population dynamics with stochastic gene expression and cell cycle variability. (a) Illustration of a growing population as a stochastic branching process with stochastic interdivision times. Each cell expresses two identical but noninteracting reporters (green and red) that are partitioned randomly at cell division. Red and green cells express more molecules of either reporter, which indicates intrinsic variability between cells. Yellow cells express similar levels of reporter molecules, but vary in their absolute amounts at different cell cycle stages, which constitutes extrinsic variability. A snapshot of the population (blue dashed box) quantifies the cell-to-cell variability across the population. A lineage (red path) quantifies variability over time and tracks an isolated cell over successive cell divisions by randomly selecting one of the daughter cells. (b) Simulated trajectories of cell age and stochastic protein expression of two identical reporters on a branched tree. Line colour indicates reporter expression in the same cell. (c) Cell age and reporter expression of an isolated cell lineage. (d) Comparison of distributions obtained from lineages and population snapshots. Simulations of the reactions (21) assume $k_{0}=10, k_{m}=1, k_{s}=10$ for each reporter and lognormal-distributed division times with unit mean and standard deviation. 

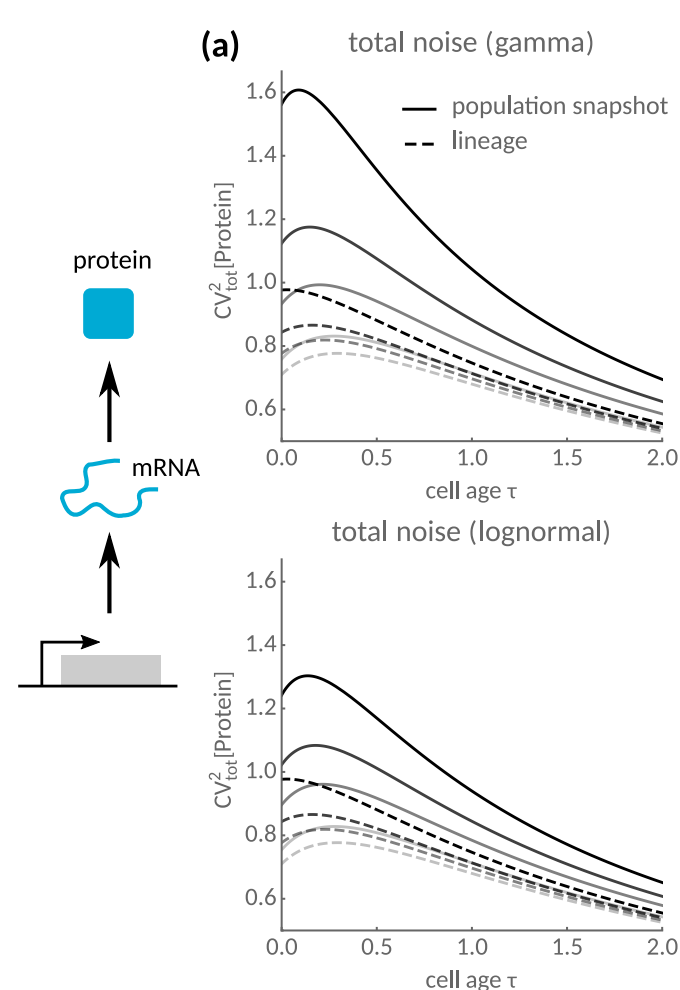

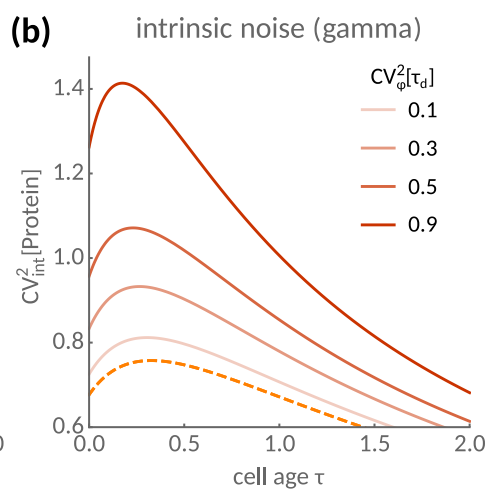

intrinsic noise (lognormal)

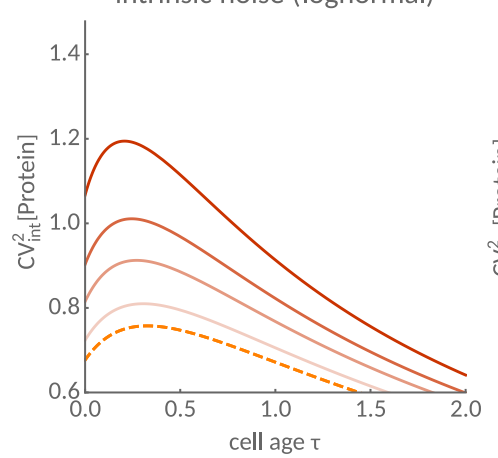

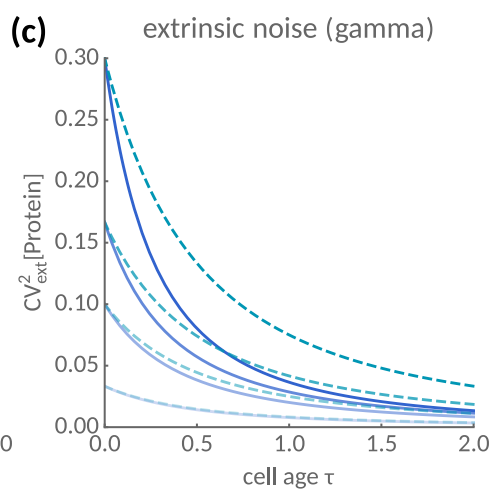

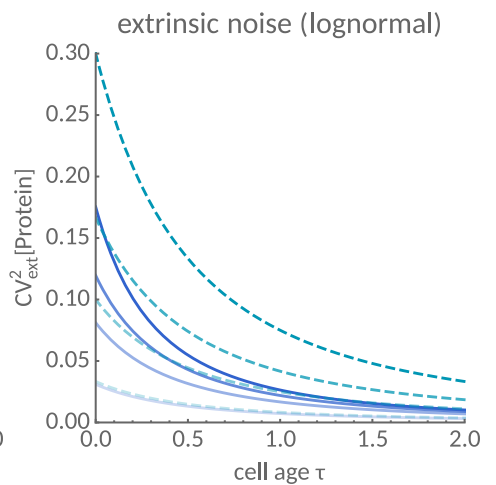

FIG. 2. Intrinsic and extrinsic noise propagation over the cell cycle. (a) Total noise as a function of cell age $\tau$ with gamma (top) and log-normal-distributed (bottom) interdivision times. Population snapshot statistics (solid) are compared to lineages (dashed lines). Noise is monotonic for large cell cycle fluctuations $\mathrm{CV}^{2}\left[\tau_{d}\right]$ in lineages but not in snapshots. (b) Intrinsic noise peaks as a function of cell age and increases with cell cycle fluctuations in populations but not in lineages. (c) Extrinsic noise is lower in the population than in lineages. Parameters are $k_{0}=1, b=100$ and division time distributions assume unit mean. 

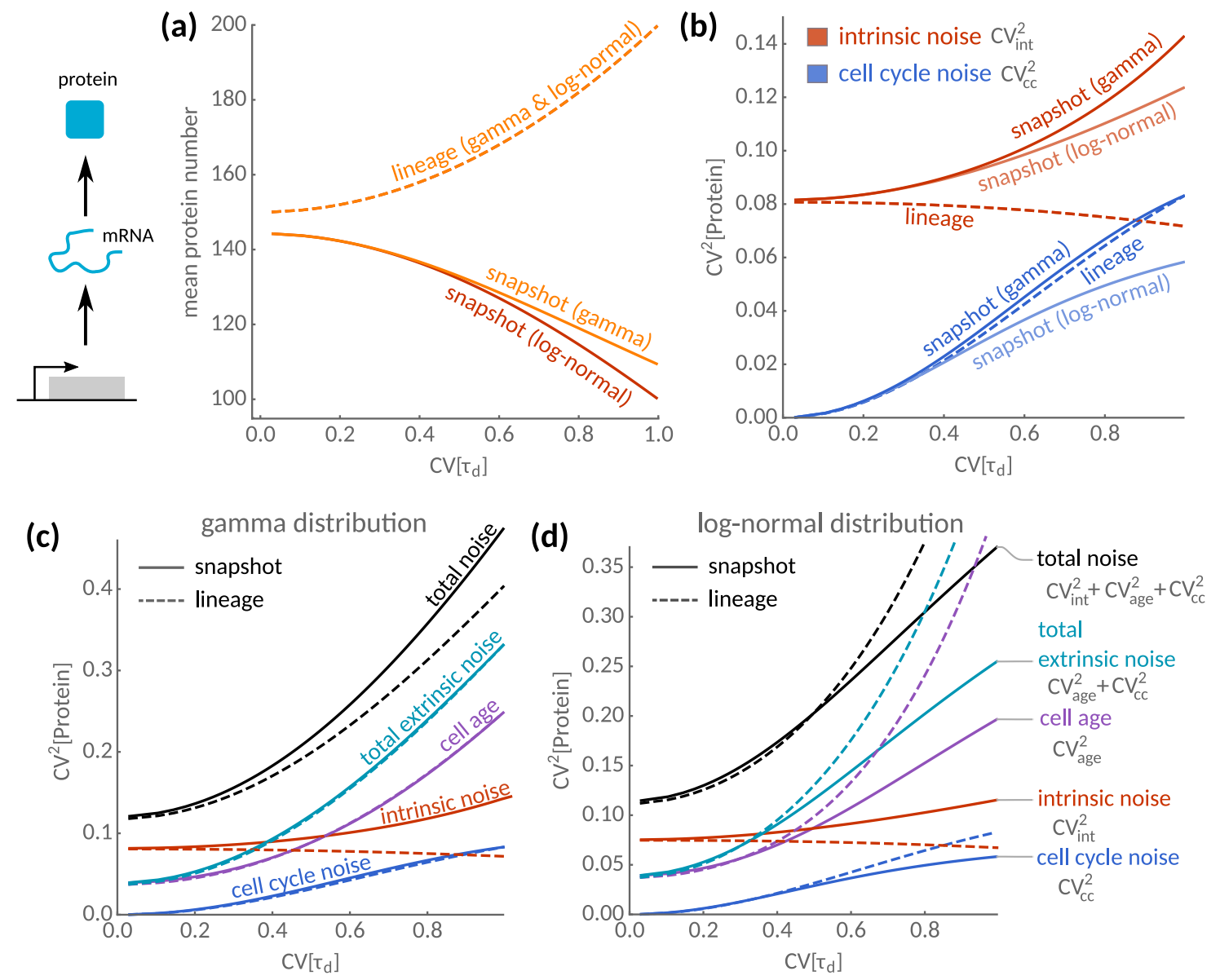

FIG. 3. Statistics of population snapshots and isolated lineages for cells of unknown age. (a) Mean protein number as a function of the cell cycle variations $\mathrm{CV}\left[\tau_{d}\right]$ in lineages (dashed) and snapshots (solid lines). For lineages, the mean protein number increases with cell cycle variability and is independent of the division time distribution. In snapshots, the mean decreases with cell cycle variability with a rate that depends on higher moments of the distribution. The predictions for gamma- and log-normal distributed interdivision times is shown. (b) Sensitivity of intrinsic and extrinsic noise sources to cell cycle fluctuations. Intrinsic noise (red lines) increases in lineages but decreases in snapshots consistent with the dependence of the respective means shown in (a). The transmitted cell cycle noise (blue lines) shows a similar dependence on cell-cycle variability in lineages and snapshots for the gamma-distribution, but is lower in snapshots for the log-normal distribution. (c) Total noise (black lines) broken down into individual noise components for the gamma-distribution. Transmitted cell cycle noise and the uncertainty due to distributed cell ages (purple lines) contribute to the total extrinsic noise (teal). (d) The corresponding break-down for the log-normal distribution. Parameters are $k_{0}=10$ and $b=10$ and $E_{\varphi}\left[\tau_{d}\right]=1$. 

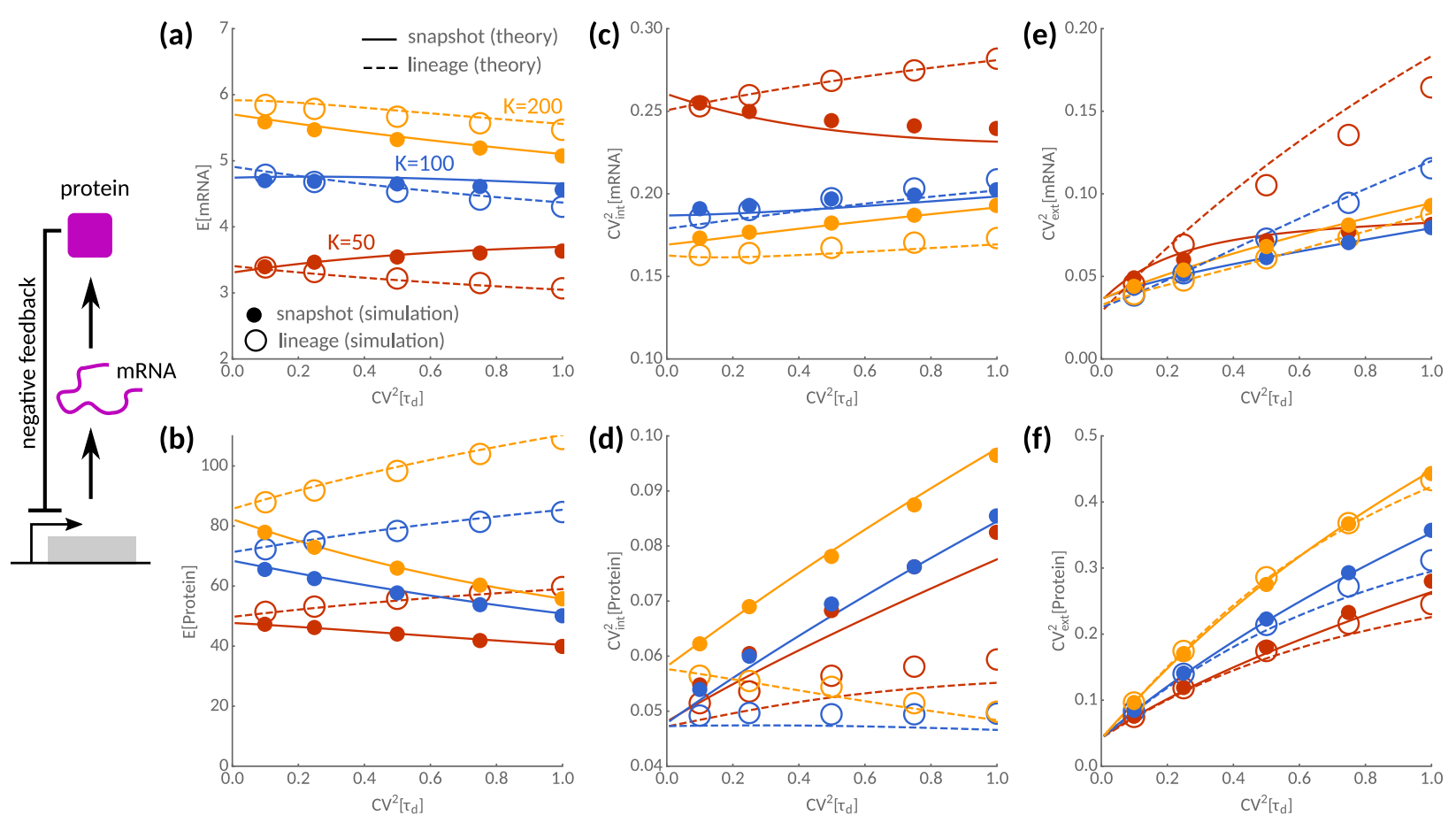

FIG. 4. Noise decomposition of a negative feedback circuit. Sensitivities to cell cycle noise $\mathrm{CV}^{2}\left[\tau_{d}\right]$ of mean, intrinsic and extrinsic noise contributions are shown for weak (yellow, $K=200$ ), moderate (blue, $K=100$ ) and strong feedback (red, $K=50$ ). Predictions by the linear noise approximation (solid lines) are in good qualitative agreement with stochastic simulations (dots). (a) In lineages, the mean mRNA number always decreases with cell cycle variability while this is not true in snapshots for moderate to high feedback. (b) In contrast, protein levels always increase in lineages but decrease in snapshots. (c) The corresponding intrinsic noise profiles of mRNAs typically increase with cell cycle noise except in snapshots with strong feedback. (d) Intrinsic noise of proteins always increases with cell cycle noise in snapshots but not in lineages. (e,f) Total extrinsic noise increases with cell cycle variability for mRNAs and proteins. However, strong feedback may significantly reduce extrinsic noise in snapshots compared to lineages. Deviations between the approximation (lines) and the simulations (dots) are most pronounced for strong feedback. Parameters are $k_{0}=10, k_{m}=1, k_{s}=10$ and unit-mean interdivision times. 

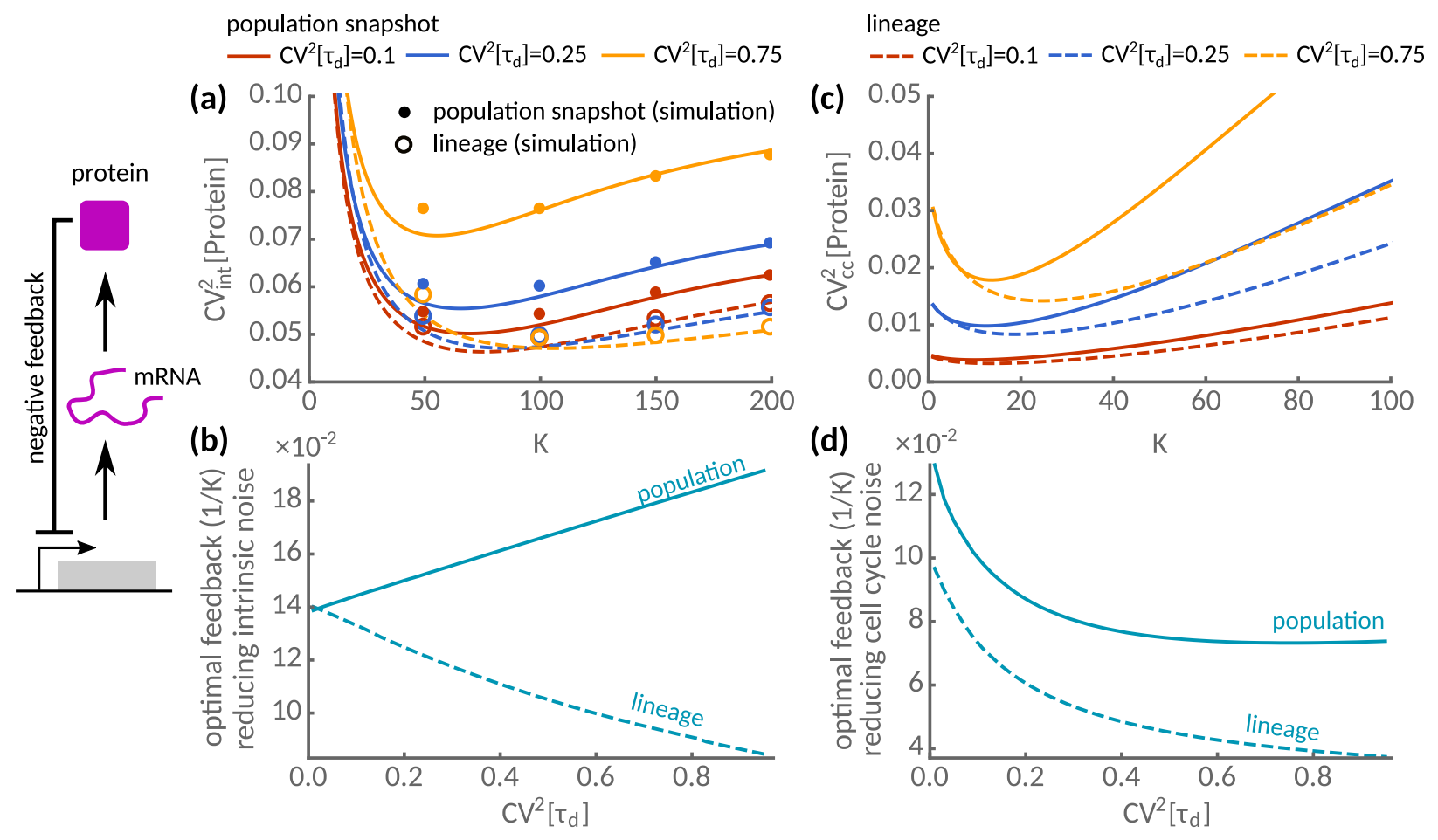

FIG. 5. Feedback strategies for noise suppression in lineages and populations. Intrinsic and extrinsic noise statistics of negative autoregulatory feedback circuit are shown as a function of $K$, the inverse feedback strength, for three different levels of cell cycle noise $\mathrm{CV}^{2}\left[\tau_{d}\right]=0.1$ (red), 0.25 (blue) and 0.75 (yellow). (a) Intrinsic noise exhibits a minimum as a function of the repression strength both in lineage (dashed) and in snapshot statistics (solid lines). The predictions obtained using the linear noise approximation (lines) are in good agreement with exact stochastic simulations using the First-Division Algorithm ${ }^{31}$ (dots for population, open circles for lineages). (b) Optimal feedback strength $(1 / K)$ to minimise intrinsic noise is shown. The feedback strength increases with interdivision time noise in lineages but decreases in population snapshots. (c) The transmitted cell cycle noise shows a minimum in dependence of the repression strength both in lineage (dashed) and in snapshot statistics (solid lines). (d) The optimal feedback strength to minimise transmitted cell cycle noise decreases with division time noise both in lineages and to a lesser extent in the population. Parameters as in Fig. 4 